\title{
Problemi di curvatura su di una varietà quasi hermitiana.
}

\author{
G. B. RIzzA (a Parma) $\left(^{*}\right)(* *)$
}

Sunto. - Su di una rarietà quasi hermitiana $V$, la considerazione di aloune medie di curvature

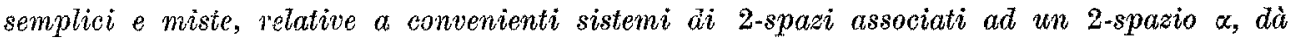
informasioni sulla curvatura semplice $K_{\alpha}$, sulla media $\frac{1}{2}\left(K_{\alpha}+K_{\tilde{\alpha}}\right)$ e sulla curvatura mista $\chi_{\alpha \tilde{\alpha}}$ $\left(\tilde{\alpha}=J_{\alpha}\right)$. Due delle medie accennate riescono nulle; le altre danno lwogo a relazioni di dipendenza lineare. Oonsiderate per la varietà $V$ ipotesi di isotropia in media e di J-isotropia si ottengono teoremi del tipo di Schur. Altri risultati si stabiliscono nel caso hähleriano.

Summary. - Let $V$ be an almost Hermite space, $\alpha$ a tangent 2-dimensional space of $V$; five sys. tems of tangent 2-spaces of $V$ are considered, suitably related to $\alpha$. The sectional curvature $K_{\alpha}$, the mean value $\frac{1}{2}\left(K_{\alpha}+K_{\tilde{\alpha}}\right)$, and the bisectional curvature $\chi_{\alpha \tilde{\alpha}}(\tilde{\alpha}=J \alpha)$ are expressed in terms of mean values of sectional and bisectional curvatures, evalued over the above mentioned systems. Two of the mean values vanish; linear relationships hold among the other ones. Schur-like theorems are proven under the assumption that $\nabla$ be isotropic in mean or $J$-isotropic. Further results are obtained for the case that $V$ be a Kähler space.

\section{1. - Introduzione.}

Sulle curvature delle sezioni 2-dimensionali delle varietà kähleriane sono noti nella letteratura diversi risultati $\left(^{1}\right)$. Questo lavoro è uno studio delle curvature ora accennate nel caso più generale delle varietà quasi hermitiane.

Ai risultati si perviene sviluppando una idea, rivelatasi già promettente nel caso kähleriano $\left({ }^{2}\right)$, quella cioè di associare al 2 -spazio $\alpha$ in considerazione opportuni sistemi di 2-spazi caratteristici o a prodotto hermitiano reale.

Precisamente, si considerano qui $\mathrm{i}$ sistemi $\Sigma_{\alpha}, \stackrel{\circ}{\alpha}_{\alpha}$ di 2 -spazi caratteristici $\left({ }^{3}\right)$ ed i sistemi $\dot{\Sigma}_{\alpha}, \hat{\Sigma}_{\alpha}, \hat{\Sigma}_{\alpha}$ di 2 -spazi a prodotto hermitiano reale, convenientemente parametrizzati (nn. 4, $5,6,7,8$ ), e si valutano le medie $\mu_{\Sigma}$ e $M_{\Sigma}$ delle curvature semplici e miste dei 2 -spazi dei sistemi $\Sigma$. ì utile introdurre anche la media $m_{\alpha}$ delle curvature miste $\chi_{\alpha \omega}$ al variare di $\omega$ in $\Sigma_{\alpha}$.

(*) Entrata in Redazione il 25 novembre 1971.

(**) Lavoro eseguito con contributo del C.N.R. nell'ambito del Gruppo Nazionale Strutture Algebriche, Geometriche e loro Applicazioni.

I primi risultati sono stati annunciati in una riunione di Gruppo a Bologna (dicembre 1970) $\mathrm{e}$ in una conferenza tenuta all'Ist. Mat. Univ. Perugia (aprile 1971).

(1) Si vedano p. es. i nn. [4], [11], [8] della bibliografia posta in fine.

(2) G. B. Rrzza [8], n. 3, p. 84 .

(3) Il primo di questi ha un ruolo importante anche in altri miei lavori ([6], [8]). 
Ciò premesso, le medie $M_{\widehat{\Sigma}_{\alpha}}, M_{\bar{\Sigma}_{\alpha}}$ sono nulle (teoremi $\mathbf{T}_{1}, \mathbf{T}_{2}$, nn. 13, 14). Le altre medie relative $a \hat{\Sigma}_{\alpha}, \hat{\Sigma}_{\alpha}, \hat{\Sigma}_{\alpha}$ si esprimono linearmente nelle medie $\mu, M, m$ relative ai sistemi $\infty^{1}, \Sigma_{\alpha}, \dot{\Sigma}_{\alpha}$ e nelle curvature semplici e miste $K_{\alpha}, K_{\tilde{\alpha}}, \chi_{\alpha \tilde{\alpha}}$, essendo $\tilde{\alpha}$ il 2-spazio che corrisponde ad $\alpha$ nell'isomorfismo fondamentale $J$ della struttura quasi com. plessa (nn. 12, 13, 14).

Seguono molti risultati. La curvatura mista $\chi_{\alpha \tilde{\alpha}}$ è combinazione lineare delle medie $\mu, M$ relative ai sistemi $\Sigma_{\alpha}, \dot{\Sigma}_{\omega}$ (teorema $\mathbf{T}_{4}$ n. 17). Teoremi dello stesso tipo, che fanno intervenire anche $\mathrm{i}$ sistemi $\hat{\Sigma}_{\alpha}, \hat{\Sigma}_{\alpha}$, sussistono per la curvatura semplice $K_{\alpha}$ e per la media $\frac{1}{2}\left(K_{\alpha}+K_{\tilde{\alpha}}\right)$ (teoremi $\mathbf{T}_{5}, \mathbf{T}_{6}$, n. 17 ).

Si prova inoltre che le medie $\mu, M$ relative ai sistemi $\Sigma_{\alpha}, \dot{\Sigma}_{\alpha}$ sono linearmente $d i-$ pendenti, i coefficienti essendo funzioni della deviazione caratteristica di $\alpha$ (teorema $\mathbf{T}_{3}$, n. 17). Altre relazioni di dipendenza lineare tra medie sono indicate al $\mathbf{n} .18$.

Tutte le medie considerate si possono esprimere mediante le curvature (semplici e miste) relative ad $\alpha$, $\tilde{\alpha}$ e ad un numero finito di 2 -spazi di $\Sigma_{\alpha}$ e $\dot{\Sigma}_{\alpha}$ (n. 15) ed hanno natura $J$-invariante ad eccezione di $m_{\alpha}$ e di $\mu_{\hat{\Sigma}_{x}}$ (n. 16).

Il n. 19 è dedicato ai casi particolari di $\alpha$ caratteristico ovvero a prodotto hermitiano reale.

Nelle ipotesi di isotropia in media e di $J$-isotropia in un punto della varietà (qui considerate per la prima volta), si stabiliscono alcuni risultati (teoremi $\mathbf{T}_{11}, \mathbf{T}_{12}, \mathbf{T}_{13} ;$ n. 20), dai quali derivano teoremi del tipo di Sorrun (teoremi $\mathbf{T}_{14}, \mathbf{T}_{15}$; corollario $\mathbf{C}_{\mathbf{1}}$; n. 22).

Infine, nel caso particolare delle varietà kähleriane, si ottengono altri risultati (teoremi $\mathbf{T}_{16}, \mathbf{T}_{17}, \mathbf{T}_{18}, \mathbf{T}_{19} ;$ n. 23) e si ritrovano alcuni teoremi noti. Sia l'isotropia in media che la $J$-isotropia si riducono in questo caso all'isotropia ordinaria.

\section{2. - Struttura quasi complessa. 2-spazi caratteristici.}

Sia $V$ una varietà a struttura quasi complessa $\left({ }^{4}\right), \underset{\mathbf{R}}{\operatorname{dim}} V=2 n(n \geqslant 2), \underset{\mathbf{R}}{\operatorname{cl} V} V=r$ $(r \geqslant 3), x$ un punto di $V, \tau$ lo spazio vettoriale tangente ad $x$ in $V, \tau_{*}$ lo spazio vettoriale duale.

Indicato con $\mathcal{H}$ il campo tensoriale misto, $\underset{\mathbf{R}}{\mathrm{c}} \mathfrak{H}=s(1 \leqslant s \leqslant r-1)$, che determina la struttura di $V$, sia $h \in \tau_{*} \otimes \tau$ il corrispondente tensore nel punto $x$ di $V$ e $J$ l'isomorfismo anti-involutorio definito in $\tau$ dalla struttura quasi complessa. Se $u, v, w$ sono vettori di $\tau$, si pone $\tilde{u}=J u, \tilde{v}=J v, \widetilde{w}=J w, \ldots$.

亡 immediato estendere $J$ ai bivettori, ponendo $J(u \wedge v)=\tilde{u} \wedge \tilde{v}$. Sui bivettori $J$ ha carattere involutorio.

Sia ora $\alpha$ il 2-spazio vettoriale di $\tau$ definito dal bivettore non nullo $u \wedge v$. Si indica con $\tilde{\alpha}=J \alpha$ il 2 -spazio vettoriale definito da $\tilde{u} \wedge \tilde{v}\left(^{5}\right)$.

(') Per le nozioni fondamentali sulle varietà quasi complesse si veda p. es. K. YANo [11], ch. 5; S. Koba yashi - K. Nomizu [4], II, ch. 9, n, 2. Vedi anche G. B. Rizza [9], n. 2.

(5) $\mathrm{E}$ utile talora indicare $\alpha$ semplicemente con $w . \tilde{\alpha}$ può quindi indicarsi con $\tilde{u} \tilde{v}$. 
Ciò premesso un 2-spazio vettoriale $\alpha$ di $\tau$ si dice caratteristico, se e solo se risulta $\alpha=\tilde{\alpha}\left({ }^{6}\right)$. Ogni vettore non nullo $u$ di $\tau$ appartiene ad uno e ad un solo 2 -spazio caratteristico; precisamente appartiene al 2 -spazio $\alpha$ definito dal bivettore $u \wedge \tilde{u}$.

Più in generale, utilizzando multivettori, si perviene alla definizione di $k$-spazi caratteristici $(2 \leqslant k \leqslant 2 n)$.

\section{3. - Struttura quasi-hermitiana. 2-spazi p.h.r.}

Si assume ora che $V$ sia dotata di struttura quasi hermitiana di classe non inferiore a $2\left(^{7}\right) . V$ è quindi una varietà riemanniana e la metrica $g$ di $V\left(2 \leqslant{ }_{\mathbf{R}} g \leqslant r-1\right)$ è adattata alla struttura quasi complessa. Risulta cioè

$$
u \cdot v=\tilde{u} \cdot \tilde{v} \quad u, v \in \tau
$$

il punto denotando il prodotto scalare. In particolare i vettori $u, \tilde{u}$ sono ortogonali. Si definisce poi il prodotto hermitiano ${ }^{8}$ )

$$
u * v=\frac{1}{2}(u \cdot v+i(u \cdot \tilde{v}))
$$

Intervengono nel seguito i 2 -spazi vettoriali di $\tau$ a prodotto hermitiano reale (brevemente 2-spazi p.h.r.). Essi sono individuati dai bivettori non nulli $u \wedge v$, soddisfacenti alla condizione $u \cdot \tilde{v}=0\left({ }^{\circ}\right)$.

Conviene ricordare che, condizione necessaria e sufficiente perchè $\alpha$ sia p.h.r. $\grave{e}$ che $\alpha$ ed $\tilde{\alpha}$ siano ortogonali.

\section{4. - Sistema $\Sigma_{\alpha}$.}

Sia $\alpha$ un 2-spazio non caratteristico $(\alpha \neq \tilde{\alpha})$. Si denoti con $\Sigma_{\alpha}$ il sistema $\infty^{1}$ dei 2-spazi caratteristici aventi un 1-spazio in comune con $\alpha\left({ }^{10}\right)$.

Se $u, v$ sono vettori unitari e ortogonali di $\alpha$ si considerino i vettori

$$
z=u \cos \theta+v \sin \theta \quad 0 \leqslant \theta<2 \pi .
$$

(6) Il termine "2-spazio vettoriale caratteristico" equivale alla locuzione inglese "holo. morphic section $"$.

(7) Sulle varietà quasi hermitiane si veda p. es. K. Yaxo [11], ch. 9; S. Kobayashi . K. Nomizu [4], ch. 9, n. 4.

(8) Sulle metriche hermitiane, euclidee, simplettiche vedi E. Martinedu [5].

$\left.{ }^{9}\right)$ G. B. Rrzza [6], p. 669; [7], p. 5. I risultati di questi lavori hanno carattere pun. tuale; essi valgono quindi anche nel caso quasi hermitiano (vedi [7], nota $\left({ }^{3}\right)$, p. 4). Uno studio generale sugli spazi p.h.r. si trova nel lavoro [2] di M. BRUNI.

(10) G. B. RizzA [6], p. 664 . 
I bivettori $z \wedge \tilde{z}$ individuano i 2 -spazi di $\Sigma_{\alpha}$. E noto che l'angolo che i 2 -spazi $\omega$ di $\Sigma_{x}$ formano con $\alpha$ è costante. Quest'angolo prende nome di deviazione caratteristica di $\alpha$ e si denota con $\delta_{\alpha}$ : Risulta

$$
\cos \delta_{\alpha}=\tilde{u} \cdot v
$$

Previo eventuale scambio dei vettori $u$, $v$, si può supporre $0 \leqslant \delta_{\alpha} \leqslant \pi / 2$. Risulta allora in particolare $\delta_{\alpha}=0, \delta_{\alpha}=\pi / 2$, se e solo se $\alpha$ è caratteristico, a prodotto hermitiano reale, rispettivamente.

\section{5. - Sistema $\stackrel{*}{\Sigma}_{\alpha}$.}

Se $\alpha$ non è caratteristico $(\alpha \neq \tilde{\alpha})$, si consideri lo spazio vettoriale $A$ generato da $\alpha$ e $\tilde{\alpha} . A$ è un 4-spazio vettoriale caratteristico ( $\left.{ }^{11}\right)$.

Ciò premesso si consideri il sistema $\dot{\Sigma}_{\alpha}$ costituito dagli $\infty^{1} 2$-spazi a prodotto hermitiano reale, aventi un 1-spazio in comune con $\alpha$ ed un 1-spazio in comune con $\tilde{\alpha}$.

I 2-spazi di $\dot{\Sigma}_{\alpha}$ sono definiti dai bivettori $r \wedge \tilde{s}$ con

$$
r=u \cos \varphi+v \sin \varphi, \quad \tilde{s}=-\tilde{u} \sin \varphi+\tilde{v} \cos \varphi,
$$

e $0 \leqslant \varphi<2 \pi$.

Infatti il 2-spazio $\varrho$ individuato da $r \wedge \tilde{s}$ ha un 1-spazio in comune sia con $\alpha$ che con $\tilde{\alpha}$ (definiti da $r$ e da $\tilde{s}$ rispettivamente). D'altronde sia $r$ che $\tilde{s}$ sono ortogonali ai vettori $\tilde{r}$ ed $s$; dunque $\varrho$ è ortogonale a $\varrho$ e pertanto è un 2-spazio p.h.r. (n. 3). In definitiva risulta $\varrho \in \dot{\Sigma}_{\alpha}$.

Inversamente, sia $\varrho$ un 2 -spazio di $\dot{\Sigma}_{x}$ ed

$$
r=u \cos \varphi+v \sin \varphi, \quad \tilde{t}=\tilde{u} \cos \psi+\tilde{v} \sin \psi,
$$

due vettori unitari di $\varrho$ appartenenti ad $\alpha$ e ad $\tilde{\alpha}$ rispettivamente. Poichè $\varrho$ è p.h.r. risulta $r \cdot t=0$ (n. 3 ). Ne segue $\cos (\varphi-\psi)=0$ da cui $\tilde{t}= \pm \tilde{s}$. In conclusione $\varrho$ può essere definito dal bivettore $r \wedge \tilde{s}$, con $r, \tilde{s}$ dati dalla (5).

6. - Sistema $\stackrel{\circ}{\Sigma_{\alpha}}$.

Sempre nell'ipotesi $\alpha \neq \tilde{\alpha}$, si consideri il sistema $\stackrel{\circ}{\Sigma}_{\alpha}$, constituto dagli $\infty^{2} 2-s p a z i$ caratteristici del 4-spazio caratteristico $A$ definito da $\propto$ e $\tilde{\alpha}{ }^{(12)}$.

(11) E ovvio che i vettori $u, v, \tilde{u}, \tilde{v}$ generano $A$; di più essi risultano indipendenti. Infatti, i bivettori $u \wedge \tilde{u}, v \wedge \tilde{v}$ danno luogo a 2 -spazi caratteristici e questi non hanno 1-spazi in comune, altrimenti, in base ad una osservazione del n. 2, coinciderebbero; ma allora sarebbe $\alpha=\tilde{\alpha}$.

(12) Ogni $2 p$-spazio caratteristico $\omega(p \in N)$ può riguardarsi come un $p$-spazio vettoriale su. $\boldsymbol{C}$, ponendo $\left(a^{\prime}+i a^{\prime \prime}\right) u=a^{\prime} u+a^{\prime \prime} \tilde{u}\left(a^{\prime}, a^{\prime \prime} \in \boldsymbol{R} ; u \in \omega\right)$. 
È utile per il seguito l'osservazione

$\mathbf{0}_{1}-$ Gli elementi di $\dot{L}_{\alpha}^{\circ}$ sono $i 2$-spazi caratteristici associati, nel senso indicato al $n .4$, ai 2-spazi p.h.r. di $\dot{\Sigma}_{\alpha}$. Ciò̀

$$
\stackrel{\circ}{\Sigma}_{\alpha}=\left\{\omega \in \Sigma_{\varrho}: \varrho \in \dot{\Sigma}_{\alpha}\right\} .
$$

Scelto arbitrariamente $\varrho$ in $\dot{\Sigma}_{\alpha}$, dalla (5) segue subito che $\varrho$ e $\tilde{g}$ generano $A$; perciò ogni $\omega \in \Sigma_{\varrho}$ appartiene ad $A$ e quindi a $\Sigma_{\alpha}$.

Inversamente occorre provare che ogni 2 -spazio caratteristico $\omega^{\prime}$ di $\stackrel{R}{\alpha}_{\alpha}$ puó riguardarsi come un 2-spazio $\omega$ associato ad un 2-spazio $\varrho$ di $\dot{L}_{\alpha}$.

Si noti che, in virtù di una osservazione del n. 2 , l'elemento generico $\omega^{\prime}$ dì $\stackrel{\circ}{\alpha}_{\alpha}$ può individuarsi assegnando ad arbitrio un vettore unitario $x$ di $A$. Sia cioè

$$
x=a_{1} u+a_{2} \tilde{u}+a_{3} v+a_{4} \tilde{v} \quad\left(a_{j} \in \boldsymbol{R}\right)
$$

con

$$
a_{1}^{2}+a_{2}^{2}+a_{3}^{2}+a_{4}^{2}-2\left(a_{1} a_{4}-a_{2} a_{3}\right) \cos \delta_{\alpha}=1
$$

D'altra parte, sia $x^{\prime}$ il più generale vettore unitario appartenente ad un 2 -spazio caratteristico $\omega$ di $\Sigma_{\varrho}$, con $\varrho$ arbitrario 2 -spazio p.h.r. di $\dot{\Sigma}_{\alpha}$. Risulta

$$
x^{\prime}=w^{\prime} \cos \tau+\widetilde{w}^{\prime} \sin \tau
$$

dove

$$
w^{\prime}=\lambda r+\mu \tilde{s},
$$

con $r, \tilde{s}$ dati dalla (5) e $\lambda, \mu$ soddisfacenti alla condizione

$$
\lambda^{2}+\mu^{2}-2 \lambda \mu \cos \delta_{\alpha}=1
$$

Ciò premesso, se, fissato arbitrariamente un vettore unitario $x$ di $A$, esiste un vettore $x^{\prime}$ del tipo ora indicato coincidente con $x$, in base alla osservazione del n. 2 sopra richiamata, risulta $\omega^{\prime}=\omega$ e ciò prova quanto si voleva.

Dalle (6), (8) segue subito che la condizione $x=x^{\prime}$ si traduce nel sistema

$$
\begin{aligned}
& a_{1}=\lambda \cos \varphi \cos \tau+\mu \sin \varphi \sin \tau \\
& a_{2}=-\mu \sin \varphi \cos \tau+\lambda \cos \varphi \sin \tau \\
& a_{3}=\lambda \sin \varphi \cos \tau-\mu \cos \varphi \sin \tau \\
& a_{4}=\mu \cos \varphi \cos \tau+\lambda \sin \varphi \sin \tau
\end{aligned}
$$

nelle variabili $\lambda, \mu, \varphi, \tau$. 
Dal sistema (11) discendono le equazioni

$$
\begin{aligned}
& a_{1}^{2}+a_{2}^{2}+a_{3}^{2}+a_{4}^{2}=\lambda^{2}+\mu^{2}, \quad a_{1} a_{4}-a_{2} a_{3}=\lambda \mu, \\
& \frac{a_{2}+a_{3}}{a_{1}-a_{4}}=\operatorname{tg}(\varphi+\tau), \quad \frac{a_{3}-a_{2}}{a_{1}+a_{4}}=\operatorname{tg}(\varphi-\tau),
\end{aligned}
$$

che permettono di ricavare $\lambda, \mu, \varphi, \tau$.

Dalla condizione (7) segue naturalmente la (10).

Si verifica infine che $\mathrm{i}$ valori trovati costituiscono tna soluzione del sistema

$$
\begin{aligned}
& a_{1}+a_{4}=(\lambda+\mu) \cos (\varphi-\tau) \\
& a_{1}-a_{4}=(\lambda-\mu) \cos (\varphi+\tau) \\
& a_{2}+a_{3}=(\lambda-\mu) \sin (\varphi+\tau) \\
& a_{3}-a_{2}=(\lambda+\mu) \sin (\varphi-\tau)
\end{aligned}
$$

e quindi del sistema (11).

È utile per il seguito notare che la condizione (10) equivale ad assumere

$$
\begin{aligned}
& \lambda=\frac{1}{2}\left(\frac{\sin \Theta}{\sin \delta_{\alpha} / 2}+\frac{\cos \Theta}{\cos \delta_{\alpha} / 2}\right)=\left(\sin \delta_{\alpha}\right)^{-1} \sin \left(\Theta+\frac{\delta_{\alpha}}{2}\right) \\
& \left.\mu=\frac{1}{2}\left(\frac{\sin \Theta}{\sin \delta_{\alpha} / 2}-\frac{\cos \Theta}{\cos \delta_{\alpha} / 2}\right)=\left(\sin \delta_{\alpha}\right)^{-1} \sin \left(\Theta-\frac{\delta_{\alpha}}{2}\right){ }^{13}\right) .
\end{aligned}
$$

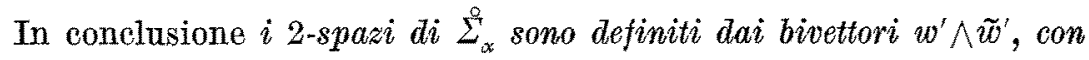

$$
w^{\prime}=\left(\sin \delta_{\alpha}\right)^{-1}\left(r \sin \left(\Theta+\frac{\delta_{\alpha}}{2}\right)+\tilde{s} \sin \left(\Theta-\frac{\delta_{\alpha}}{2}\right)\right)
$$

ed $r, \tilde{s}$ dati dalla (5).

\section{7. - Sistema $\hat{\Sigma}_{\alpha}$.}

Sia $\alpha$ un qualunque 2 -spazio vettoriale di $\tau$, non caratteristico $(\alpha \neq \tilde{\alpha})$. Si consideri il sistema $\widehat{\Sigma}_{\alpha}$, costituito dagli $\infty^{2} 2-s p a z i$ p.h.r. di A, aventi in comune con $\propto$ un 1-spazio.

E utile osservare che 2 -spazi $d i \hat{\Sigma}_{\alpha}$ sono individuati dai bivettori $r \wedge y$, con

$$
y=\left(\operatorname{tg} \delta_{\alpha}\right)^{-1}(r \cos \psi-\tilde{r} \sin \psi)+\left(\sin \delta_{\alpha}\right)^{-1}(s \sin \psi+\tilde{s} \cos \psi),
$$

$e d r, \tilde{s} d a t i d a(5)$

(13) Interpretata la (10) come equazione di una ellisse, basta operare la riduzione a forma canonica per ottenere la (12). 
Invero è immediato riconoscere che i vettori $r, s, \tilde{r}, \tilde{s}$ come $u, v, \tilde{u}, \tilde{v}$, costituiscono una base in $A$ (n. 5).

Ciò premesso, i 2 -spazi p.h.r. di $A$, aventi in comune con $\alpha$ l'1-spazio definito da $r$, sono individuati dai bivettori $r \wedge y$ con $y=b_{1} r+b_{2} \tilde{r}+b_{3} s+b_{4} \tilde{s}$ soddisfacenti alle condizioni

$$
y \cdot y=1, \quad r \cdot y=0, \quad r \cdot \tilde{y}=0
$$

Queste si traducono in

$$
\begin{aligned}
& b_{1}^{2}+b_{2}^{2}+b_{3}^{2}+b_{4}^{3}-2\left(b_{1} b_{4}-b_{2} b_{3}\right) \cos \delta_{\alpha}=1, \\
& b_{1}=b_{4} \cos \delta_{\alpha}, \quad b_{2}=-b_{3} \cos \delta_{\alpha} .
\end{aligned}
$$

Dalle ultime due, sostituendo nella precedente, si trae

$$
\left(b_{3}^{2}+b_{4}^{2}\right) \sin ^{2} \delta_{\alpha}=1
$$

onde può scegliersi

$$
b_{3}=\left(\sin \delta_{\alpha}\right)^{-1} \sin \psi, \quad b_{4}=\left(\sin \delta_{\alpha}\right)^{-1} \cos \psi,
$$

e quindi

$$
b_{1}=\left(\operatorname{tg} \delta_{\alpha}\right)^{-1} \cos \psi, \quad b_{2}=-\left(\operatorname{tg} \delta_{\alpha}\right)^{-1} \sin \psi
$$

Si perviene così alla (14).

\section{8. - Sistema $\stackrel{\Delta}{\Sigma_{\alpha}}$.}

Si consideri ora, sempre nell'ipotesi $\alpha \neq \tilde{\alpha}$, il sistema $\hat{\Sigma}_{\alpha}$, costituito dai 2-spazi a prodotto hermitiano reale di $A$. Gli elementi di $\hat{\Sigma}_{\alpha}$ sono $\infty^{3}$. Invero, rappresentando $A$ come spazio affine, le giaciture dei piani per l'origine corrispondenti ai 2 -spazi di $\hat{\Sigma}_{\alpha}$ costituiscono un complesso lineare non speciale in uno spazio proiettivo di dimensione 3.

Da un risultato generale di M. BruNI (14) risulta che i 2 -spazi di $\hat{\Sigma}_{\alpha}$ si possono ottenere a partire dalle coppie di 2 -spazi caratteristici di $A$ ortogonali.

Precisamente, se $\omega_{1}, \omega_{2}$ sono 2 -spazi di $\stackrel{\digamma}{\alpha}_{\alpha}$ ortogonali, risultano p.h.r. i 2 -spazi definiti dai bivettori $x_{1} \wedge x_{2}$ con $x_{i} \in \omega_{3}$. Al variare della coppia ortogonale $\omega_{1}, \omega_{2}$ in $A$, si ottengono tutti i 2 -spazi di $\hat{\Sigma}_{\alpha}$ :

Ciò premesso, considerati i vettori $r_{j}, \tilde{s}_{j}$ ottenuti dalla (5) del n. 5 per $\varphi=\varphi_{j}$,

(14) M. BRuxi [2], p. 283. 
a norma dell'osservazione al termine del $\mathrm{n} .6$, i bivettori $w_{1} \wedge \widetilde{w}_{1}, w_{2} \wedge \widetilde{w}_{2}$ con

$$
w_{i}=\left(\sin \delta_{\alpha}\right)^{-1}\left(r_{j} \sin \left(\Theta_{j}+\frac{\delta_{\alpha}}{2}\right)+\tilde{s}_{j} \sin \left(\Theta_{j}-\frac{\delta_{\alpha}}{2}\right)\right),
$$

definiscono una coppia di 2-spazi caratteristici $\omega_{1}, \omega_{2}$ in $A$.

La condizione di ortogonalità tra $\omega_{1}$ ed $\omega_{2}$ si traduce immediatamente nella

$$
w_{1} \cdot w_{2}=w_{1} \cdot \tilde{w}_{2}=0
$$

Tenuto conto che

$$
\begin{array}{ll}
r_{1} \cdot r_{2}=\tilde{s}_{1} \cdot \tilde{s}_{2}=\cos \left(\varphi_{1}-\varphi_{2}\right) ; & r_{1} \cdot \tilde{s}_{2}=\tilde{s}_{1} \cdot r_{2}=-\cos \left(\varphi_{1}-\varphi_{2}\right) \cos \delta_{x}, \\
r_{1} \cdot g_{2}=-\tilde{s}_{1} \cdot \tilde{r}_{2}=\sin \left(\varphi_{1}-\varphi_{2}\right) ; & r_{1} \cdot \tilde{r}_{2}=-\tilde{s}_{1} \cdot s_{2}=\sin \left(\varphi_{1}-\varphi_{2}\right) \cos \delta_{x} .
\end{array}
$$

Si riconosee con calcolo diretto che la (16) equivale a

$$
\cos \left(\varphi_{1}-\varphi_{2}\right) \cos \left(\Theta_{1}-\Theta_{2}\right)=\sin \left(\varphi_{1}-\varphi_{2}\right) \cos \left(\Theta_{1}+\Theta_{2}\right)=0
$$

Non è difficile ormai concludere che le $\infty^{2}$ coppie di 2-spazi caratteristici ortogonali di $A$ si ottengono quando i parametri $\varphi_{1}, \varphi_{2}, \Theta_{1}, \Theta_{2}$ soddisfano alla condizione

$$
\varphi_{1}, \varphi_{2} \text { arbitrari, } \quad \Theta_{1}=\frac{\pi}{2}, \quad \Theta_{2}=0,
$$

ovvero alla condizione

$$
\varphi_{1}=\varphi_{2}, \quad \Theta_{1}=\Theta_{2}+\frac{\pi}{2}
$$

Posto infine

$$
x_{j}=w_{j} \cos \tau_{j}+\tilde{w}_{j} \sin \tau_{j},
$$

con $w_{1}, w_{12}$ soddisfacenti ad una delle condizioni (18), in virtù di una osservazione precedente $i$ bivettori $x_{1} \wedge x_{2}$ definiscono i 2-spazi di $\hat{\Sigma}_{\alpha}$, oioè $i 2$-spazi p.h.r. di A.

Conviene ancora notare che, nella costruzione indicata, ogni 2-spazio p.h.r. di $\hat{\Sigma}_{\alpha}$ viene ottenuto $\infty^{1}$ volte, in corrispondenza cioè alle $\infty^{1}$ sue basi ortonormali.

\section{9. - Curvatura semplice e mista.}

Conviene ora ricordare le definizioni di curvatura (semplice e mista relative ai 2-spazi di $\tau\left({ }^{15}\right)$.

(15) La nozione di curvatura semplice è classica (vedi p. es. J. A. Schouxen [10], p. 153); quella di curvatura mista è dovuta a E. BoMptanI ([1], p. 130). I termini inglesi sono rispettivamente "sectional, bisectional curvature". 
Siano $\alpha, \beta$ due 2-spazi di $\tau$, definiti rispettivamente dai bivettori $u \wedge v$ ed $x \wedge y$ e si denoti con $R \in \tau_{*} \otimes \tau_{*} \otimes \tau_{*} \otimes \tau_{*}$ il tensore di curvatura di RIEMANN nel punto $x$ di $V$. Ciò premesso, la curvatura di $\alpha$ e la curvatura mista di $\alpha$ e $\beta$ sono date rispetivamente da

$$
\begin{aligned}
\Pi_{\alpha} & =-\frac{R(u, v, u, v)}{\operatorname{mis}^{2} u \operatorname{mis}^{2} v \sin ^{2} u v} \\
\chi_{\alpha \beta} & =-\frac{R(u, v, x, y)}{\operatorname{mis} u \operatorname{mis} v \operatorname{mis} x \operatorname{mis} y \sin u v \sin x y}
\end{aligned}
$$

dove $\operatorname{mis} w=\sqrt[+]{w \cdot w}$.

Le definizioni $(20),(21)$ di curvatura (semplice e mista) dipendono solo dai 2-spazi in considerazione e non dalla scelta di una base in essi. Sono tuttavia utili nel seguito notazioni del tipo $K_{w v}$ e $\chi_{w v, x y}$ per indicare rispettivamente $K_{\alpha}$ e $\chi_{\alpha \beta}$.

Se in $\alpha$ e $\beta$ si assumono basi ortonormali, le (20), (21) divengono semplicemente

$$
\begin{aligned}
& K_{\alpha}=-R(u, v, u, v), \\
& \chi_{\alpha \beta}=-R(u, v, x, y) .
\end{aligned}
$$

È pure ovvio che $\chi_{\alpha \alpha}=K_{\alpha}$.

In particolare, quando i 2-spazi $\alpha$ e $\beta$ sono caratteristici (n. 2), si parla di ourvatura caratteristica (semplice e mista) ${ }^{\left({ }^{16}\right)}$.

Conviene per il seguito segnalare la relazione

$$
\chi_{\alpha \tilde{\alpha}}=\chi_{u \tilde{u}, \tilde{u} \tilde{v}}-\chi_{w \tilde{v}, \varepsilon \tilde{u}} \sin ^{2} \delta_{\alpha},
$$

dove $u, v$ è una base ortonormale di $a$. Essa deriva direttamente dalla prima identità di BIANCEI per il tensore $R\left({ }^{17}\right)$, tenendo presentile definizioni $(20,(21)$ e la (4) del n. 4.

Infine, sia $\Sigma$ uno qualunque dei sistemi di 2-spazi introdotti ai n. 4, 5, 6, 7, 8 . Si denotino, rispettivamente, con $\mu_{\Sigma}$ e con $M_{\Sigma}$ le medie (in relazione alla parametrizzazione di $\Sigma$ indicata) delle curvature sempliei $K_{\alpha}$ e miste $\chi_{\alpha \beta}$ al variare dei 2-spazi $\alpha$ e $\beta$ in $\Sigma$.

I successivi numeri $10,11,12,13,14$ sono dedicati alla valutazione di queste medie.

10. - Medie $\mu_{\Sigma_{\alpha}}$ e $M_{\Sigma_{\alpha}}$.

Si procede anzitutto alla valutazione delle medie delle curvature caratteristiche (semplici e miste) relative al sistema $\Sigma_{\alpha}$ del $n, 4$.

${ }^{\left({ }^{16}\right)}$ Per la nozione di curvatura caratteristica (in inglese "holomorphie sectional cur. vature y si veda p. es. K. YANo - S. Bocriner [12], p, 127. La nozione di curvatura caratteristica mista (in inglese "holomorphic bisectional curvature") è stata introdotta e utiliz. zata per la prima volta nel mio lavoro [8] del 1959. Ciò sembra essere sfuggito ad alcuni Autori (vedi p. es. S. I. Goloberg - S. Kobay Ashi [3], p. 226).

(17) Vedi p. es. K. NomizU - S. KobaYashi [4], I, pp. 198-201. 
Siano $z_{1}, z_{2}$ i vettori ottenuti ponendo nella (3) $\theta=\theta_{1}, \theta_{2}$ rispettivamente ed $\omega_{1}$, $\omega_{2}$ i 2 -spazi di $\Sigma_{\alpha}$ definiti dai bivettori $z_{1} \wedge \tilde{z}_{1}, z_{2} \wedge \tilde{z}_{2}$. Tenuta presente la (22) e le classiche identità relative al tensore di RIEManN $R\left({ }^{18}\right)$, si ha

$$
\begin{aligned}
-\chi_{\omega_{2} \omega_{2}}=R\left(\tilde{z}_{1} \tilde{z}_{1} z_{2} \tilde{z}_{2}\right) & =\left\{R(u \tilde{u} u \tilde{u}) \cos ^{2} \theta_{2}+R(u \tilde{u} \tilde{v}) \sin ^{2} \theta_{2}\right\} \cos ^{2} \theta_{1} \\
& +\{R(u \tilde{u} u \tilde{v})+R(u \tilde{u} \tilde{u})\} \sin \left(\theta_{1}+\theta_{2}\right) \cos \theta_{1} \cos \theta_{2} \\
& +\frac{1}{4}\{R(u \tilde{v} u \tilde{v})+2 R(u \tilde{v} \tilde{u})+R(v \tilde{u} v \tilde{u})\} \sin 2 \theta_{1} \sin 2 \theta_{2} \\
& +\{R(v \tilde{v} v \tilde{u})+R(v \tilde{v} u \tilde{v})\} \sin \left(\theta_{1}+\theta_{2}\right) \sin \theta_{1} \sin \theta_{2} \\
& +\left\{R(v \tilde{v} u \tilde{u}) \cos ^{2} \theta_{2}+R(v \tilde{v} v \tilde{v}) \sin ^{2} \theta_{2}\right\} \sin ^{2} \theta_{1} .
\end{aligned}
$$

Ciò premesso, integrando $\chi_{\omega_{1} \omega_{3}}$ da 0 a $2 \pi$ rispetto alle variabili $\theta_{1}$ e $\theta_{2}$ e dividendo per $(2 \pi)^{2}$ si ottiene subito la media delle curvature miste di $\Sigma_{\alpha}$

$$
M_{\Sigma_{\kappa}}=-\frac{1}{4}\{R(u \tilde{u} u \tilde{u})+2 R(u \tilde{u} v \tilde{v})+R(v \tilde{v} v \tilde{v})\} .
$$

Si consideri ora in particolare il caso $\theta_{1}=\theta_{2}=\theta$; risulta allora $\omega_{1}=\omega_{2}=\omega$ (n. 4) e $\chi_{\omega_{1} \omega_{3}}=K_{\omega}$ (n. 9). Pertanto, ponendo nella (25) $\theta_{1}=\theta_{2}=\theta$, integrando da 0 a $2 \pi$ rispetto a $\theta$ e dividendo per $2 \pi$, si ottiene la media delle curvature sempliei di $\Sigma_{\alpha}$

$$
\begin{aligned}
\mu_{\Sigma_{\tilde{v}}}= & -\frac{1}{8}\{3 R(u \tilde{u} u \tilde{u})+2 R(u \tilde{u} v \tilde{v})+3 R(v \tilde{v} v \tilde{v})\} \\
& -\frac{1}{8}\{R(u \tilde{v} u \tilde{v})+2 R(u \tilde{v} v \tilde{u})+R(v \tilde{u} v \tilde{u})\} .
\end{aligned}
$$

Tenuto conto delle (20), (21) e della uguaglianza

$$
\sin ^{2} u \tilde{v}=\sin u \tilde{v} \sin v \tilde{u}=\sin ^{2} v \tilde{u}=\sin ^{2} \delta_{\alpha}\left({ }^{19}\right),
$$

le medie $\mu_{\Sigma_{\alpha}}$ e $M_{\Sigma_{\alpha}}$ delle curvature semplici e miste dei 2-spazi di $\Sigma_{\alpha}$ possono esprimersi in modo semplice mediante curvature di 2 -spazi particolari; precisamente sussistono le relazioni

$$
\begin{aligned}
& \mu_{\Sigma_{\alpha}}=\frac{1}{8}\left(3 K_{u \tilde{u}}+2 \chi_{u \tilde{u}, v \tilde{v}}+3 K_{v \tilde{v}}\right)+\frac{1}{8}\left(K_{u \tilde{v}}+2 \chi_{u \tilde{v}, \tilde{u}}+K_{q \tilde{u}}\right) \sin ^{2} \delta_{\alpha}, \\
& M_{\Sigma_{\alpha}}=\frac{1}{4}\left(K_{u \tilde{u}}+2 \chi_{u \tilde{u}, v \tilde{v}}+K_{v \tilde{v}}\right) .
\end{aligned}
$$

Conviene per il seguito introdurre anche la media $m_{\alpha}$ delle curvature miste $\chi_{\alpha \omega}$ al variare di $\omega$ in $\Sigma_{\alpha}$. Tenute presenti la (23) e la (3) del n. 4, un calcolo analogo ai precedenti conduce alla relazione

$$
m_{x}=\frac{1}{2}\left(\chi_{u v, u \tilde{u}}+\chi_{u v, v \tilde{v}}\right) .
$$

(18) Tedi p. es. K. Nomizu - S. Kobayashi [4], I, pp. 198-201. Si scriverà semplicemente $R(u v x y)$ in luogo di $R(u, v, x, y)$.

(19) Dalla (1) segue subito che per due vettori unitari $u, v$ (eventnalmente coincidenti) si ha $\sin u \tilde{v}=\sin v \tilde{u}$. In particolare, se $u, v$ sono ortogonali, sussiste la (4), cioè $\cos \delta_{\alpha}=\cos \tilde{u} v$. 
11. - Medie $\mu_{\dot{\Sigma}_{\alpha}}$ e $M_{\dot{\Sigma}_{\alpha}}$.

Con riferimento al sistema $\infty^{1}$ di 2 -spazi p.h.r. $\dot{\Sigma}_{\alpha}$, introdotto al n. 5, si considerano ora le medie delle curvature (semplici e miste) $\mu_{\dot{\Sigma}_{\alpha}}$ e $M_{\dot{\Sigma}_{\alpha}}$.

Siano $r_{1}, \tilde{s}_{1}$ ed $r_{2}, \tilde{s}_{2}$ i vettori ottenuti ponendo nella (5) $\varphi=\varphi_{1}, \varphi_{2}$ rispettivamente, e $\varrho_{1}, \varrho_{2}$ i 2 -spazi di $\dot{\Sigma}_{\alpha}$ definiti da $r_{1} \wedge \tilde{s}_{1}, r_{2} \wedge \tilde{s}_{2}$. Poichè $r_{j}, s_{j}(j=1,2)$ è una base ortonormale di $\alpha$ (n. 5), dalla (4) segue $\cos \delta_{\alpha}=r_{i} \cdot \tilde{s}_{j}=\cos r_{j} \tilde{s}_{j}$. Tenuto conto della (23) e delle proprietà di $R$, si perviene a

$$
\begin{aligned}
-\chi_{Q_{1} e_{2}} \sin ^{2} \delta_{\alpha}=R\left(r_{1} \tilde{s}_{1} r_{2} \tilde{s}_{2}\right) & =\left\{R(u \tilde{v} u \tilde{v}) \cos ^{2} \varphi_{2}-R(u \tilde{v} v \tilde{u}) \sin ^{2} \varphi_{2}\right\} \cos ^{2} \varphi_{1} \\
& -\{R(u \tilde{u} u \tilde{v})-R(v \tilde{v} u \tilde{v})\} \sin \left(\varphi_{1}+\varphi_{2}\right) \cos \varphi_{1} \cos \varphi_{2} \\
& +\frac{1}{4}\{R(u \tilde{u} u \tilde{u})-2 R(u \tilde{u} v \tilde{v})+R(v \tilde{v} v \tilde{v})\} \sin 2 \varphi_{1} \sin 2 \varphi_{2} \\
& -\{R(v \tilde{v} v \tilde{u})-R(u \tilde{u} v \tilde{u})\} \sin \left(\varphi_{1}+\varphi_{2}\right) \sin \varphi_{1} \sin \varphi_{2} \\
& +\left\{R(v \tilde{u} \tilde{u}) \sin ^{2} \varphi_{2}-R(v \tilde{u} u \tilde{v}) \cos ^{2} \varphi_{2}\right\} \sin ^{2} \varphi_{1} .
\end{aligned}
$$

Dalla (32), integrando da 0 a $2 \pi$ rispetto alle variabili $\varphi_{1}$ e $\varphi_{2}$ e dividendo per $(2 \pi)^{2}$ si ottiene la media delle curvature miste di $\dot{\Sigma}_{\alpha}$

$$
M_{\Sigma_{\alpha}}=-\frac{1}{4}\left(\sin \delta_{\alpha}\right)^{-2}\{R(u \tilde{v} u \tilde{v})-2 R(u \tilde{v} v \tilde{u})+R(v \tilde{u} v \tilde{u})\}
$$

Se si pone nella (32) $\varphi_{1}=\varphi_{2}=\varphi$, risulta $\varrho_{1}=\varrho_{2}=\varrho$ e $\chi_{e_{12}}=K_{\varrho^{2}}$. Integrando poi da 0 a $2 \pi$ rispetto a $\varphi$ e dividendo per $2 \pi$ si ottiene la media delle curvature semplici di $\dot{\Sigma}_{\star}^{*}$

$$
\begin{aligned}
\mu_{\Sigma_{\alpha}}= & -\frac{1}{8}(\sin \delta)^{-2}\{R(u \tilde{u} u \tilde{u})-2 R(u \tilde{u} v \tilde{v})+R(v \tilde{v} v \tilde{v})\} \\
& -\frac{1}{8}\left(\sin \delta_{\alpha}\right)^{-2}\{3 R(u \tilde{v} u \tilde{v})-2 R(u \tilde{v} v \tilde{u})+3 R(v \tilde{u} \tilde{u})\} .
\end{aligned}
$$

Tenuto conto ora delle (20), (21) e della (28) del $\mathrm{n} .10$, le medie $\mu_{\Sigma_{\alpha}}$ e $M_{\Sigma_{\alpha}}$ delle ourvature semplici e miste dei $2-s p a z i$ di $\Sigma_{\alpha}$ risultano espresse in modo semplice in termini di curvature particolari; precisamente sussistono le relazioni

$$
\begin{aligned}
& \mu_{\dot{\Sigma}_{\alpha}}=\frac{1}{8}\left(\sin \delta_{\alpha}\right)^{-2}\left(K_{u \tilde{u}}-2 \chi_{u \tilde{u}, v \tilde{v}}+K_{u \tilde{v}}\right)+\frac{1}{8}\left(3 K_{u \tilde{v}}-2 \chi_{u \tilde{v}, v \tilde{u}}+3 K_{v \tilde{u}}\right), \\
& M_{\Sigma_{\alpha}}=\frac{1}{4}\left(K_{u \tilde{v}}-2 \chi_{u \tilde{v}, v \tilde{u}}+K_{v \tilde{u}}\right) .
\end{aligned}
$$

12. - Medie $\mu \stackrel{\circ}{\Sigma_{\alpha}}$ e $M \stackrel{\circ}{\Sigma}_{\alpha}$.

Si considera ora il sistema $\Sigma_{\alpha}^{\circ}$ del n. 6, e le medie $\mu \stackrel{\circ}{\Sigma_{\alpha}} M \Sigma_{\alpha}^{n}$ delle curvature caratteristiche (semplici e miste) ad esso relative.

Siano $w_{1}, w_{2}$ i vettori ottenuti ponendo nella (13) $r=r_{1}, r_{2} ; s=s_{1}, s_{2} ; \Theta=\Theta_{1}, \Theta_{2}$ rispettivamente, ed $\omega_{1}, \omega_{2}$ i 2 -spazi di $\stackrel{\circ}{\alpha}_{\alpha}$ definiti da $w_{1} \wedge \tilde{w}_{1}, w_{2} \wedge \tilde{w}_{2}$. Tenute presenti 
le proprietà elementari di $R$ si ottiene subito

$$
\begin{aligned}
& -\sin ^{4} \delta_{\alpha} \chi_{\omega_{1} \omega_{2}}=\sin ^{4} \delta_{\alpha} R\left(w_{1} \tilde{w}_{1} w_{2} \tilde{w}_{2}\right)= \\
& \quad=\left\{R\left(r_{1} \tilde{r}_{1} r_{2} \tilde{r}_{2}\right) \sin ^{2}\left(\Theta_{2}+\frac{\delta_{\alpha}}{2}\right)+R\left(r_{1} \tilde{r}_{1} s_{2} \tilde{s}_{2}\right) \sin ^{2}\left(\Theta_{2}-\frac{\delta_{\alpha}}{2}\right)\right\} \sin ^{2}\left(\Theta_{1}+\frac{\delta_{\alpha}}{2}\right) \\
& \quad-\left\{R\left(r_{1} \tilde{r}_{1} r_{2} s_{2}\right)+R\left(r_{1} \tilde{r}_{1} \tilde{r}_{2} \tilde{s}_{2}\right)\right\} \sin ^{2}\left(\Theta_{1}+\frac{\delta_{\alpha}}{2}\right) s_{2} \\
& \quad-\left\{R\left(s_{1} \tilde{s}_{1} r_{2} \tilde{s}_{2}\right)+R\left(s_{1} \tilde{s}_{1} \tilde{r}_{2} \tilde{s}_{2}\right)\right\} \sin ^{2}\left(\Theta_{1}-\frac{\delta_{\alpha}}{2}\right) S_{2} \\
& \quad+\left\{R\left(r_{1} s_{1} r_{2} s_{2}\right)+R\left(r_{1} s_{1} \tilde{r}_{2} \tilde{s}_{2}\right)+R\left(\tilde{r}_{1} \tilde{s}_{1} r_{2} s_{2}\right)+R\left(\tilde{r}_{1} \tilde{s}_{1} \tilde{r}_{2} \tilde{s}_{2}\right)\right\} S_{1} S_{2} \\
& -\left\{R\left(\tilde{r}_{1} \tilde{s}_{1} s_{2} \tilde{s}_{2}\right)+R\left(r_{1} s_{1} s_{2} \tilde{s}_{2}\right)\right\} \sin ^{2}\left(\Theta_{2}-\frac{\delta_{\alpha}}{2}\right) S_{1} \\
& \quad-\left\{R\left(\tilde{r}_{1} \tilde{s}_{1} r_{2} \tilde{r}_{2}\right)+R\left(r_{1} s_{1} r_{2} \tilde{r}_{2}\right)\right\} \sin ^{2}\left(\Theta_{2}+\frac{\delta_{\alpha}}{2}\right) S_{1} \\
& \quad+\left\{R\left(s_{1} \tilde{s}_{1} r_{2} \tilde{r}_{2}\right) \sin ^{2}\left(\Theta_{2}+\frac{\delta_{\alpha}}{2}\right)+R\left(s_{1} \tilde{s}_{1} s_{2} \tilde{s}_{2}\right) \sin ^{2}\left(\Theta_{2}-\frac{\delta_{\alpha}}{2}\right)\right\} \sin ^{2}\left(\Theta_{1}-\frac{\delta_{\alpha}}{2}\right)
\end{aligned}
$$

dove

$$
S_{j}=\sin \left(\Theta_{j}+\frac{\delta_{\alpha}}{2}\right) \sin \left(\Theta_{j}-\frac{\delta_{\alpha}}{2}\right)
$$

Dalla (37) discende poi

$$
\begin{aligned}
-\frac{4 \sin ^{4} \delta_{\alpha}}{(2 \pi)^{2}} \int_{0}^{2 \pi} d \Theta_{2} \int_{0}^{2 \pi} \gamma_{\omega_{1} \omega_{2}} d \Theta_{1}=\left\{R\left(r_{1} \tilde{r}_{1} r_{2} \tilde{r}_{2}\right)+R\left(s_{1} \tilde{s}_{1} s_{2} \tilde{s}_{2}\right)+R\left(r_{1} \tilde{r}_{1} s_{2} \tilde{s}_{2}\right)+R\left(s_{1} \tilde{s}_{1} r_{2} \tilde{r}_{2}\right)\right\} \\
-\left\{R\left(r_{1} s_{1} r_{2} \tilde{r}_{2}\right)+R\left(r_{1} s_{1} s_{2} \tilde{s}_{2}\right)+R\left(r_{1} \tilde{r}_{1} r_{2} s_{2}\right)+R\left(s_{1} \tilde{s}_{1} r_{2} s_{2}\right)\right\} \cos \delta_{\alpha} \\
-\left\{R\left(\tilde{r}_{1} \tilde{s}_{1} r_{2} \tilde{r}_{2}\right)+R\left(\tilde{r}_{1} \tilde{s}_{1} s_{2} \tilde{s}_{2}\right)+R\left(r_{1} \tilde{r}_{1} \tilde{r}_{2} \tilde{s}_{2}\right)+R\left(s_{1} \tilde{s}_{1} \tilde{r}_{2} \tilde{s}_{2}\right)\right\} \cos \delta_{\alpha} \\
+ \\
+\left\{R\left(r_{1} s_{1} r_{2} s_{2}\right)+R\left(r_{1} s_{1} \tilde{r}_{2} \tilde{s}_{2}\right)+R\left(\tilde{r}_{1} \tilde{s}_{1} r_{2} s_{2}\right)+R\left(\tilde{r}_{1} \tilde{s}_{1} \tilde{r}_{2} \tilde{s}_{2}\right)\right\} \cos ^{2} \delta_{\alpha} .
\end{aligned}
$$

Occorre ora tener conto che $r_{1}, \tilde{s}_{1}, r_{2}, \tilde{s}_{2}$ sono espressi dalla (5) del n. 5 con $\varphi=\varphi_{1}$, $\varphi_{2}$ rispettivamente, e procedere all'integrazione nelle variabili $\varphi_{i}$. Tenute presenti le (22), (23) del n. 9 e il significato dei simboli $M_{\Sigma_{\alpha}}, m_{\alpha},($ n. 10) si perviene alla relazione:

$$
M_{\Sigma_{\alpha}}=\left(\sin \delta_{\alpha}\right)^{-4}\left\{M_{\Sigma_{\alpha}}-\left(m_{\alpha}+m_{\tilde{\alpha}}\right) \cos \delta_{\alpha}+\frac{1}{4}\left(K_{\alpha}+2 \chi_{\alpha \tilde{\alpha}}+K_{\tilde{\alpha}}\right) \cos ^{2} \delta_{\alpha}\right\} .
$$

Si consideri ora in particolare il caso $r_{1}=r_{2}=r ; s_{1}=s_{2}=s ; \Theta_{1}=\Theta_{2}=\Theta$; si ha allora $w_{1}=w_{2}=w$ e quindi $\omega_{1}=\omega_{2}=\omega$ mentre, $\chi_{\omega_{1} \omega_{2}}=K_{w}$ (n. 9). Dalla (37), nelle 
ipotesi attuali segue subito

$$
\begin{aligned}
-\frac{8 \sin ^{4} \delta_{\alpha}}{2 \pi} \int_{0}^{2 \pi} K_{\omega} d \Theta & =3(R(r \tilde{r} r \tilde{r})+R(s \tilde{s} \tilde{s}))+2 R(r \tilde{r} s \tilde{s})\left(1+2 \cos ^{2} \delta_{\alpha}\right) \\
& -6(R(r s r \tilde{r})+R(r s s \tilde{s})+R(\tilde{r} \tilde{s} r \tilde{r})+R(\tilde{r} \tilde{s} s \tilde{s})) \cos \delta_{\alpha} \\
& +(R(r s r s)+2 R(r s \tilde{r} \tilde{s})+R(\tilde{r} \tilde{s} \tilde{r} \tilde{s}))\left(1+2 \cos ^{2} \delta_{\alpha}\right) .
\end{aligned}
$$

Occorre ora tener conto della (5) del $n$. 5 e integrare nella variabile $\varphi$. Si noti anzitutto che, posto nella (25) $\theta_{1}=\varphi, \theta_{2}=\varphi+\pi / 2$ si ottiene

$$
\begin{aligned}
\frac{1}{2 \pi} \int_{0}^{2 \pi} R(r \tilde{r} s \tilde{s}) d \varphi & =\frac{1}{8}(R(u \tilde{u} u \tilde{u})+6 R(u \tilde{u} v \tilde{v})+R(v \tilde{v} v \tilde{v}))- \\
& -\frac{1}{8}(R(u \tilde{v} u \tilde{v})+2 R(u \tilde{v} \tilde{u})+R(v \tilde{u} v \tilde{u}))
\end{aligned}
$$

da cui, in virtù delle $(26),(27)$ discende

$$
\frac{1}{2 \pi} \int_{0}^{2 \pi} R(\boldsymbol{r} \tilde{r} s \tilde{s}) d \varphi=\mu_{\Sigma_{\alpha}}-2 M_{\Sigma_{\alpha}}
$$

Cio premesso, tenute presenti le (22), (23) del n. 9 e il significato dei simboli $\mu_{\Sigma_{\alpha}}$, $M_{\Sigma_{\alpha}}, m_{\alpha}$, (n. 10), si perviene senza difficoltà alla relazione

$$
\begin{aligned}
\mu_{\Sigma_{\alpha}} & =\frac{1}{8}\left(\sin \delta_{\alpha}\right)^{-4}\left\{4 \mu_{\Sigma_{\alpha}} \sin ^{2} \delta_{\alpha}+4 M_{\Sigma_{\alpha}}\left(1+2 \cos ^{2} \delta_{\alpha}\right)+\right. \\
& \left.-12\left(m_{\alpha}+m_{\tilde{\alpha}}\right) \cos \delta_{\alpha}+\left(K_{\alpha}+2 \chi_{\alpha \tilde{\alpha}}+K_{\tilde{\alpha}}\right)\left(1+2 \cos ^{2} \delta_{\alpha}\right)\right\} .
\end{aligned}
$$

In conclusione, con le (40), (38) le medie delle curvature (semplici e miste) degli $\infty^{2}$ 2-spazi del sistema $\stackrel{\Sigma}{\Sigma}_{\alpha}$ possono esprimersi in funzione delle curvature (semplici e miste) dei 2-spazi $\alpha$ e $\tilde{\alpha}$ e delle medie relative ai sistemi $\infty^{2} \Sigma_{\alpha}$ e $\Sigma_{\tilde{\alpha}}$.

\section{3. - Medie $\mu_{\widehat{\Sigma}_{\alpha}}$ e $M_{\widehat{\Sigma}_{\mathfrak{x}}}$.}

Con riferimento al sistema $\infty^{2}$ di 2 -spazi p.h.r. $\hat{\Sigma}_{\alpha}$, introdotto al n. 7 , si considerano ora le medie delle curvature (semplici e miste) $\mu_{\widehat{\Sigma}_{\alpha}}$ e $M_{\widehat{\Sigma}_{\alpha}}$.

Siano $r_{1}, \tilde{s}_{1}$ e $r_{2}, \tilde{s}_{2} \mathrm{i}$ vettori ottenuti ponendo nella $(5) \varphi=\varphi_{1}, \varphi_{2}$ rispettivamente. Siano poi $y_{1}, y_{2} i$ vettori dati dalla (14) del n. 7 con $r=r_{1}, r_{2} ; s=s_{1}, s_{2} ; \psi=\psi_{1}, \psi_{2}$ rispettivamente. Si denotino infine con $\varrho_{1}, \varrho_{2}$ i 2 -spazi di $\widehat{\Sigma}_{\alpha}$ definiti dai bivettori $r_{1} \wedge y_{1}$ e $r_{2} \wedge y_{2}$.

$$
4 \text { - Annali di Matematica }
$$


Ciò premesso si stabilisce direttamente la relazione

$$
\begin{aligned}
R\left(r_{1} y_{1} r_{2} y_{2}\right) \sin ^{2} \delta_{\alpha} & =R\left(r_{1} \tilde{s}_{1} r_{2} \tilde{s}_{2}\right) \cos \psi_{1} \cos \psi_{2} \\
& +\left(R\left(r_{1} \tilde{r}_{1} r_{2} \tilde{r}_{2}\right) \cos ^{2} \delta_{\alpha}+R\left(r_{1} s_{1} r_{2} s_{2}\right)\right) \sin \psi_{1} \sin \psi_{2} \\
& -\left(R\left(r_{1} \tilde{r}_{1} r_{2} s_{2}\right)+R\left(r_{1} s_{1} r_{2} \tilde{r}_{2}\right)\right) \cos \delta_{\alpha} \sin \psi_{1} \sin \psi_{2} \\
& +\left(-R\left(r_{1} \tilde{r}_{1} r_{2} \tilde{s}_{2}\right) \cos \delta_{\alpha}+R\left(r_{1} s_{1} r_{2} \tilde{s}_{2}\right)\right) \sin \psi_{1} \cos \psi_{2} \\
& +\left(-R\left(r_{2} \tilde{r}_{2} r_{1} \tilde{s}_{1}\right) \cos \delta_{\alpha}+R\left(r_{2} s_{2} r_{1} \tilde{s}_{1}\right)\right) \cos \psi_{1} \sin \psi_{2} .
\end{aligned}
$$

Conviene qui notare esplicitamente che integrando il primo membro della (41), cioè $-\chi_{Q_{10_{2}}} \sin ^{2} \delta_{\alpha}$ (n. 9), rispetto a $\psi_{1}$ e $\psi_{2}$ si ottiene zero. Sussiste perciò il Teorema

$\mathrm{T}_{1}-$ La media $M_{\hat{\Sigma}_{\alpha}}$ delle curvature miste dei 2-spazi p.h.r. del sistema $\hat{\Sigma}_{\alpha} \dot{e}$ nulla.

Per quanto concerne le curvature semplici dei 2-spazi di $\hat{\Sigma}_{\alpha}$, si consideri in particolare $r_{1}=r_{2}=r, s_{1}=s_{2}=s$ (cioè $\left.\varphi_{1}=\varphi_{2}=\varphi\right)$ e $y_{1}=y_{2}=y \quad\left(\psi_{1}=\psi_{2}=\psi\right.$ ). Si ha quindi $\varrho_{1}=\varrho_{2}=\varrho$ e $\chi_{\varrho_{12}}=K_{\varrho}$ (n. 9 ).

Dalla (41), nell'ipotesi attuale, discende

$$
\frac{2 \sin ^{2} \delta_{\alpha}}{2 \pi} \int_{0}^{2 \pi} R(r y r y) d \psi=R(r \tilde{s} r \tilde{s})+R(r \tilde{r} r \tilde{r}) \cos ^{2} \delta_{\alpha}+R(r s r s)-2 R(r s r \tilde{r}) \cos \delta_{\alpha} .
$$

È ormai immediato, tenute presenti le (20), (21) e il significato dei simboli $\mu_{\Sigma_{\alpha}}$, $\mu_{\dot{\Sigma}_{\alpha}}, m_{\alpha}$ (n. 10, 11), pervenire alla relazione

$$
\mu_{\widehat{\Sigma}_{\alpha}}=\frac{1}{2}\left(\sin \delta_{\alpha}\right)^{-2}\left(\mu_{\tilde{\Sigma}_{\alpha}} \sin ^{2} \delta_{\alpha}+\mu_{\Sigma_{\alpha}} \cos ^{2} \delta_{\alpha}-2 m_{\alpha} \cos \delta_{\alpha}+K_{\alpha}\right) .
$$

In definitiva la media delle curvature semplici degli $\infty^{2}$ 2-spazi p.h.r. del sistema $\hat{\Sigma}_{\alpha}$ e una combinazione lineare della eurvatura del 2-spazio $\alpha$ e di medie relative ai sistemi $\infty^{1}$ $\Sigma_{\alpha}$ e $\dot{\Sigma}_{\alpha}$.

\section{4. - Medie $\mu_{\vec{\Sigma}_{\alpha}}$ e $M_{\vec{\Sigma}_{\alpha}}$.}

Si esamina ora il sistema $\hat{\Sigma}_{x}$ del n. 8 e le medie delle curvature (semplici e miste) $\mu_{\bar{\Sigma}_{\alpha}}$ e $M_{\Delta_{\alpha}}$.

In base alle conclusioni del n. 8, due 2-spazi $\varrho, \varrho^{\prime}$ appartenenti a $\hat{\Sigma}_{\alpha}$ sono definiti rispettivamente dai bivettori $x_{1} \wedge x_{2}$ e $x_{1}^{\prime} \wedge x_{2}^{\prime}$, le $x_{j}, x_{j}^{\prime}$ essendo date da espressioni del tipo (19). Queste, unitamente alle condizioni (18) ed alle ipotesi su $w_{j}, w_{j}^{\prime}$ assicurano che $x_{1}, x_{2}$ ed $x_{1}^{\prime}, x_{2}^{\prime}$ sono basi ortonormali in $\varrho$, $\varrho^{\prime}$ rispettivamente. 
Risulta subito

$$
\begin{aligned}
-\chi_{e e^{*}} & =R\left(w_{1} w_{2} w_{1}^{\prime} w_{2}^{\prime}\right) \mathrm{C}_{1} \mathrm{C}_{2}+R\left(w_{1} \tilde{w}_{2} w_{1}^{\prime} \tilde{w}_{2}^{\prime}\right) \mathrm{C}_{1} \mathrm{~s}_{2} \\
& +R\left(\tilde{w}_{1} w_{2} \tilde{w}_{1}^{\prime} w_{2}^{\prime}\right) \mathrm{s}_{1} \mathrm{C}_{2}+R\left(\tilde{w}_{1} \tilde{w}_{2} \tilde{w}_{1}^{\prime} \tilde{w}_{2}^{\prime}\right) \mathrm{s}_{1} \mathrm{~s}_{2} \\
& +\left\{R\left(w_{1} w_{2} w_{1}^{\prime} \tilde{w}_{2}^{\prime}\right) \mathrm{C}_{1}+R\left(\tilde{w}_{1} w_{2} \tilde{w}_{1}^{\prime} \tilde{w}_{2}^{\prime}\right) \mathrm{s}_{1}\right\} \cos \tau_{2} \sin \tau_{2}^{\prime} \\
& +\left\{R\left(w_{1} \tilde{w}_{2} w_{1}^{\prime} w_{2}^{\prime}\right) \mathrm{C}_{1}+R\left(\tilde{w}_{1} \tilde{w}_{2} \tilde{w}_{1}^{\prime} w_{2}^{\prime}\right) \mathrm{s}_{1}\right\} \sin \tau_{2} \cos \tau_{2}^{\prime} \\
& +\left\{R\left(w_{1} w_{2} \tilde{w}_{1}^{\prime} w_{2}^{\prime}\right) \mathrm{C}_{2}+R\left(w_{1} \tilde{w}_{2} \tilde{w}_{1}^{\prime} \tilde{w}_{2}^{\prime}\right) \mathrm{s}_{2}\right\} \cos \tau_{1} \sin \tau_{1}^{\prime} \\
& +\left\{R\left(\tilde{w}_{1} w_{2} w_{1}^{\prime} w_{2}^{\prime}\right) \mathcal{C}_{2}+R\left(\tilde{w}_{1} \tilde{w}_{2} w_{1}^{\prime} \tilde{w}_{2}^{\prime}\right) \mathrm{s}_{2}\right\} \sin \tau_{1} \cos \tau_{1}^{\prime} \\
& +\left\{R\left(\tilde{w}_{1} w_{2} w_{1}^{\prime} \tilde{w}_{2}^{\prime}\right) \cos \tau_{2} \sin \tau_{2}^{\prime}+R\left(\tilde{w}_{1} \tilde{w}_{2} w_{1}^{\prime} w_{2}^{\prime}\right) \sin \tau_{2} \cos \tau_{2}^{\prime}\right\} \sin \tau_{1} \cos \tau_{1}^{\prime} \\
& +\left\{R\left(w_{1} w_{2} \tilde{w}_{1}^{\prime} \tilde{w}_{2}^{\prime}\right) \cos \tau_{2} \sin \tau_{2}^{\prime}+R\left(w_{1} \tilde{w}_{2} \tilde{w}_{1}^{\prime} w_{2}^{\prime}\right) \sin \tau_{2} \cos \tau_{2}^{\prime}\right\} \cos \tau_{1} \sin \tau_{1}^{\prime}
\end{aligned}
$$

dove

$$
S_{j}=\sin \tau_{j} \sin \tau_{j}^{\prime}, \quad \mathrm{C}_{j}=\cos \tau_{j} \cos \tau_{j}^{\prime} .
$$

Conviene notare esplicitamente che integrando il primo membro della (43) rispetto alle variabili $\tau_{1}, \tau_{2}, \tau_{1}^{\prime}, \tau_{2}^{\prime}$ si ottiene zero. Sussiste perció il Teorema

$\mathrm{T}_{2}-$ La media $M_{\hat{\Sigma}_{\alpha}}$ delle curvature miste dei 2-spazi p.h.r. del sistema $\stackrel{\Delta}{\alpha}_{\alpha}$ nulla.

Per esaminare il caso della curvatura semplice, occorre in particolare considerare $w_{j}^{\prime}=w_{j}$ ed $x_{j}^{\prime}=x_{j}\left(\tau_{j}^{\prime}=\tau_{j}\right)$. In queste ipotesi, dalla (43) discende

$$
\begin{aligned}
& \frac{1}{(2 \pi)^{2}} \int_{0}^{2 \pi} d \tau_{2} \int_{0}^{2 \pi} R\left(x_{1} x_{2} x_{1} x_{2}\right) d \tau_{1}= \\
& \quad=\frac{1}{4}\left\{R\left(w_{1} w_{2} w_{1} w_{2}\right)+R\left(w_{1} \tilde{w}_{2} w_{1} \tilde{w}_{2}\right)+R\left(\tilde{w}_{1} w_{2} \tilde{w}_{1} w_{2}\right)+R\left(\tilde{w}_{1} \tilde{w}_{2} \tilde{w}_{1} \tilde{w}_{2}\right)\right\}
\end{aligned}
$$

con le $w_{j}$ date dalla (15) del n. 8 e soddisfacenti ad una delle condizioni (18).

Si considera anzitutto la condizione $\left(18^{\prime}\right)$. Si ha ciò̀

$$
w_{1}=\left(\sin \delta_{\alpha}\right)^{-1} \cos \frac{\delta_{\alpha}}{2}\left(r_{1}+\tilde{s}_{1}\right), \quad w_{2}=\left(\sin \delta_{\alpha}\right)^{-1} \sin \frac{\delta_{\alpha}}{2}\left(r_{2}-\tilde{s}_{2}\right)
$$

Segue

$$
\begin{aligned}
4 \sin ^{2} \delta_{\alpha} R\left(w_{1} w_{2} w_{1} w_{2}\right) & =R\left(r_{1} r_{2} r_{1} r_{2}\right)+R\left(\tilde{s}_{1} \tilde{s}_{2} \tilde{s}_{1} \tilde{s}_{2}\right)-2 R\left(r_{1} r_{2} \tilde{s}_{1} \tilde{s}_{2}\right) \\
& +R\left(r_{1} \tilde{s}_{2} r_{1} \tilde{s}_{2}\right)+R\left(\tilde{s}_{1} r_{2} \tilde{s}_{1} r_{2}\right)-2 R\left(r_{1} \tilde{s}_{2} \tilde{s}_{1} r_{2}\right) \\
& -2\left\{R\left(r_{1} r_{2} r_{1} \tilde{s}_{2}\right)+R\left(\tilde{s}_{1} \tilde{s}_{2} \tilde{s}_{1} r_{2}\right)-R\left(r_{1} r_{2} \tilde{s}_{1} r_{2}\right)-R\left(\tilde{s}_{1} \tilde{s}_{2} r_{1} \tilde{s}_{2}\right)\right\} .
\end{aligned}
$$

dove $r_{j}, \tilde{s}_{j}$ sono dati dalla (5) per $\varphi=\varphi_{j}$.

Conviene notare che la somma dei primi tre addendi a secondo membro può scri. versi

$$
-\left(K_{\alpha}-2 \chi_{\alpha \tilde{\alpha}}+K_{\tilde{\alpha}}\right) \sin ^{2}\left(\varphi_{2}-\varphi_{1}\right)
$$


Si riconosce poi che l'integrale rispetto a $\varphi_{1}, \varphi_{2}$ della quantità entro parentesi graffe ̀̀ nullo. È ormai facile pervenire alla uguaglianza

$$
\begin{gathered}
-\frac{8 \sin ^{2} \delta_{\alpha}}{(2 \pi)^{2}} \int_{0}^{2 \pi} d \varphi_{2} \int_{0}^{2 \pi} R\left(w_{1} w_{2} w_{1} w_{2}\right) d \varphi_{1}=\left(K_{\alpha}-2 \chi_{\alpha \tilde{\tilde{u}}}+K_{\tilde{\alpha}}\right)+ \\
+\left(K_{u \tilde{u}}-2 \chi_{u \tilde{u}, v \tilde{v}}+K_{v \tilde{v}}\right)+2\left(K_{u \tilde{v}}+K_{v \tilde{u}}\right) \sin ^{2} \delta_{\alpha} .
\end{gathered}
$$

Al medesimo risultato si giunge anche partendo dagli altri addendi che figurano a secondo membro della (44).

È utile denotare con $\mu_{\hat{\Sigma}_{\alpha}}^{\prime}$ la media delle curvature $K_{\varrho}$ dei 2-spazi $\varrho$ di $\hat{\Sigma}_{\alpha}$ costruiti a partire dalle coppie di 2 -spazi caratteristici $\omega_{1}, \omega_{2}$, soddisfacenti alla condizione di ortogonalità $\left(18^{\prime}\right)$. Tenute presenti la (22) e le (44), (46) con l'osservazione che segue quest'ultima, si ottiene l'uguaglianza

$$
\mu_{\hat{\Sigma}_{\alpha}}^{\prime}=\frac{1}{8}\left(\sin \delta_{\alpha}\right)^{-2}\left\{\left(K_{\alpha}-2 \chi_{\alpha \tilde{\alpha}}+K_{\tilde{\alpha}}\right)+\left(K_{u \tilde{u}}-2 \chi_{u \tilde{u}, \tilde{v}}+K_{v \tilde{v}}\right)+2\left(K_{u \tilde{v}}+K_{v \tilde{u}}\right) \sin ^{2} \delta_{\alpha}\right\} .
$$

Si riconosee poi che, in virtù delle $(30),(36)$ e della relazione $(24)$ del n. 9 , si può scrivere

$$
\mu_{\tilde{\Sigma}_{\alpha}}^{\prime}=\frac{1}{8}\left(\sin \delta_{\alpha}\right)^{-2}\left\{K_{\alpha}-6 \gamma_{\alpha \tilde{x}}+K_{\tilde{\alpha}}+4 M_{\Sigma_{\alpha}}+8 M_{\Sigma_{\alpha}} \sin ^{2} \delta_{\alpha}\right\}
$$

Si considera ora la condizione $\left(18^{\prime \prime}\right)$. Si ha

$$
\begin{aligned}
& w_{1}=\left(\sin \delta_{\alpha}\right)^{-1}\left\{r \cos \left(\Theta+\frac{\delta_{\alpha}}{2}\right)+\tilde{s} \cos \left(\Theta-\frac{\delta_{\alpha}}{2}\right)\right\}, \\
& w_{2}=\left(\sin \delta_{\alpha}\right)^{-1}\left\{r \sin \left(\Theta+\frac{\delta_{\alpha}}{2}\right)+\tilde{s} \sin \left(\Theta-\frac{\delta_{\alpha}}{2}\right)\right\} .
\end{aligned}
$$

In virtù delle proprietà di $R$ si ottiene subito l'uguaglianza

$$
R\left(w_{1} w_{2} w_{1} w_{2}\right)+R\left(\tilde{w}_{1} \tilde{w}_{2} \tilde{w}_{1} \tilde{w}_{2}\right)=\left(\sin \delta_{\alpha}\right)^{-2}\{R(r \tilde{s} r \tilde{s})+R(\tilde{r} s \tilde{r} s)\}
$$

D'altra parte, tenuto conto della (48), non è difficile pervenire alla relazione

$$
\begin{aligned}
\frac{8 \sin ^{4} \delta_{\alpha}}{2 \pi} \int_{0}^{2 \pi} R & \left(w_{1} \tilde{w}_{2} w_{1} \tilde{w}_{2}\right) d \Theta= \\
= & \left\{\left(R(r s r s)+R(\tilde{r} \tilde{s} \tilde{r} \tilde{s})\left(\left(1+2 \sin ^{2} \delta_{\alpha}\right)+2 R(r s \tilde{s} \tilde{s}) \cos 2 \delta_{\alpha}\right\}\right.\right. \\
& +\left\{R(r \tilde{r} r \tilde{r})+R(s \tilde{s} s \tilde{s})+2 R(r \tilde{r} s \tilde{s}) \cos 2 \delta_{\alpha}\right\} \\
& -2\{R(r s r \tilde{r})+R(r s s \tilde{s})+R(\tilde{r} \tilde{s} r \tilde{r})+R(\tilde{r} \tilde{s} s \tilde{s})\} \cos \delta_{\alpha} .
\end{aligned}
$$

Il medesimo risultato si ottiene a partire da $R\left(\tilde{w}_{1} w_{2} \tilde{w}_{1} w_{2}\right)$. 
Conviene ora denotare con $\mu_{\hat{\Sigma}_{\alpha}}^{\prime \prime}$ la media analoga a $\mu_{\hat{\Sigma}_{\alpha}}^{\prime}$ ma relativa alla condizione di ortogonalità $\left(18^{\prime \prime}\right)$.

Ciò premesso, tenute presenti la $(20),(21)$ del n. 9, le (44), (49), (50) e la (39) del n. 12 e le definizioni di $\mu_{\Sigma_{\alpha}}, M_{\Sigma_{\alpha}}, m_{\alpha}, \mu_{\Sigma_{\alpha}}($ n. 10,11) si perviene a

$$
\begin{aligned}
\mu_{\tilde{\Sigma}_{\alpha}}^{n} & =\frac{1}{16}\left(\sin \delta_{\alpha}\right)^{-4}\left\{\left(K_{\alpha}+K_{\tilde{\alpha}}\right)\left(1+2 \sin ^{2} \delta_{\alpha}\right)+2 \chi_{\alpha \tilde{\alpha}} \cos 2 \delta_{\alpha}+\right. \\
& \left.+2 \mu_{\Sigma_{\alpha}}\left(1-\cos 2 \delta_{\alpha}\right)+4 M_{\Sigma_{\alpha}} \cos 2 \delta_{\alpha}-4\left(m_{\alpha}+m_{\tilde{\alpha}}\right) \cos \delta_{\alpha}+8 \mu_{\Sigma_{\alpha}} \sin ^{4} \delta_{\alpha}\right\} .
\end{aligned}
$$

E ormai possibile pervenire alla conclusione.

Dalle (47), (51) discende la relazione

$$
\begin{aligned}
\mu_{\dot{\Delta}_{\alpha}}=\frac{1}{2}\left(\mu_{\hat{\Sigma}_{\alpha}}^{2}+\mu_{\Sigma_{\alpha}}^{\prime \prime}\right) & =\frac{1}{32}\left(\sin \delta_{\alpha}\right)^{-4}\left\{\left(K_{\alpha}+K_{\tilde{\alpha}}\right)\left(1+4 \sin ^{2} \delta_{\alpha}\right)\right. \\
& +2 \chi_{\alpha \tilde{\alpha}}\left(1-8 \sin ^{2} \delta_{\alpha}\right)+4 \mu_{\Sigma_{\alpha}} \sin ^{2} \delta_{\alpha}+4 M_{\Sigma_{\alpha}} \\
& \left.+8 \mu_{\Sigma_{\alpha}} \sin ^{4} \delta_{\alpha}+16 M_{\Sigma_{\alpha}} \sin ^{4} \delta_{\alpha}-4\left(m_{\alpha}+m_{\tilde{\alpha}}\right) \cos \delta_{\alpha}\right\} .
\end{aligned}
$$

Pertanto, la media delle curvature semplici degli $\infty^{3}$ 2-spazi p.h.r. del sistema $\hat{\Sigma}_{\alpha}$ è una combinazione lineare delle curvature (semplici e miste) dei 2-spazi $\alpha$ e $\tilde{\alpha}$ e di medie relative ai sistemi $\infty^{1} \Sigma_{\alpha} e \Sigma_{\alpha}$.

15. - Sistemi finiti $\mathscr{H}_{\alpha}$ e $\mathfrak{R}_{\alpha}$.

Alle considerazioni dei numeri precedenti conviene aggiungere una osservazione.

Sia $u, v$ una base ortonormale di $\alpha(\alpha \neq \tilde{\alpha}), H_{\alpha}(u, v)$ il sistema costituito dai 2-spazi caratteristici $u \tilde{u}, v \tilde{v} ; \mathfrak{R}_{\alpha}(u, v)$ il sistema dei 2-spazi p.h.r. $u \tilde{v}, v \tilde{u}$.

Ciò premesso, risulta

$\mathbf{0}_{2}-$ Le medie relative ai sistemi $\Sigma_{\alpha}, \stackrel{\circ}{\Sigma}_{\alpha}, \dot{\Sigma}_{\alpha}, \hat{\Sigma}_{\alpha}, \vec{\Sigma}_{\alpha}$ si esprimono tutte linearmente mediante le curvature (semplici e miste) di $\alpha$ e $\tilde{\alpha}$ e dei 2-spazi saratteristici e p.h.r. appartenenti ai sistemi finiti $\mathfrak{K}_{\alpha}(u, v), \mathfrak{R}_{\alpha}(u, v)$.

Per le medie $\mu_{\Sigma_{\alpha}}, M_{\Sigma_{\alpha}}, \mu_{\Sigma_{\alpha}}, M_{\Sigma_{\alpha}}, m_{\alpha}$ il risultato segue immediatamente dalle (29), $(30),(35),(36),(31)$; di conseguenza, come appare dalle $(40),(38),(42),(52)$, esso sussiste anche per le medie $\mu_{\mathcal{E \alpha}_{\alpha}}, M_{\Sigma_{\alpha}}, \mu_{\widehat{\Sigma}_{\alpha}}, \mu_{\hat{\Sigma}_{\alpha}}$.

Conviene poi introdurre la base ortonormale bisettrice

$$
x=\frac{\sqrt{2}}{2}(u+v), \quad y=\frac{\sqrt{2}}{2}(-u+v)
$$


Un calcolo diretto conduce allora a stabilire le relazioni

$$
\begin{aligned}
& \mu_{\Sigma_{\varepsilon}}=\frac{1}{4}\left(K_{u \tilde{u}}+K_{v \tilde{v}}+K_{x \tilde{x}}+K_{y \tilde{y}}\right), \\
& \mu_{\tilde{\Sigma}_{\alpha}}=\frac{1}{4}\left(K_{\tilde{u} \tilde{v}}+K_{v \tilde{u}}+K_{x \tilde{y}}+K_{y \tilde{x}}\right) .
\end{aligned}
$$

più semplici delle (29), (35). Esse fanno intervenire soltanto curvature semplici relative al sistema in considerazione.

\section{6. - $J$-invarianza.}

Prima di procedere oltre conviene osservare che la maggior parte delle nozioni e degli enti geometrici considerati nei numeri precedenti ha natura invariante nell'isomorfismo fondamentale $J$ (n. 2).

Ad esempio, è immediato notare che, oltre al prodotto scalare (n. 3), l'isomorfismo $J$ lascia invariata la deviazione caratteristica (n. 4). (Risulta cioè $\delta_{\alpha}=\delta_{\tilde{\alpha}}$ ); di conseguenza le nozioni di 2-spazio caratteristico e di 2-spazio p.h.r. $(n .2,3)$ hanno natura invariante rispetto a $J$.

Eे facile verificare poi che $i$ sistemi di 2-spazi $\Sigma_{\alpha}, \dot{\Sigma}_{\alpha}^{\circ}, \dot{\Sigma}_{\alpha}^{*}, \Delta_{\alpha}$, introdotti nei n. 4,6, 5, 8, come pure lo spazio 4-dimensionale $A$ (n. 5), sono mutati un sè dall'isomorfismo $J$. In altri termini, si ha

$$
\Sigma_{\alpha}=\Sigma_{\tilde{\alpha}}, \quad \dot{\Sigma}_{\alpha}=\dot{\Sigma}_{\tilde{\alpha}}, \quad \dot{\Sigma}_{\alpha}^{*}=\dot{\Sigma}_{\tilde{\alpha}}^{*}, \quad \hat{\Sigma}_{\alpha}=\hat{\Sigma}_{\tilde{\alpha}} .
$$

Ciò non accade invece per il sistema $\hat{\Sigma}_{\alpha}$ del n. 7. Risulta invero in generale $\hat{\Sigma}_{\alpha} \neq \hat{\Sigma}_{\alpha}$.

E importante notare che le curvature semplici e miste (n. 9) non restano in generale invariate nell'isomorfismo $J$. Si ha ciò̀ in generale $K_{\alpha} \neq K_{\tilde{\alpha}}$ e $\chi_{\alpha \beta} \neq \chi_{\tilde{\alpha} \tilde{\beta}}$.

Tuttavia, in virtù di una osservazione precedente, per le medie introdotte ai n. 10, $12,11,14$ risulta

$$
\begin{aligned}
& \mu_{\Sigma_{\alpha}}=\mu_{\Sigma_{\tilde{\alpha}}}, \quad \mu_{\Sigma_{\alpha}}=\mu_{\Sigma_{\tilde{\alpha}}}, \quad \mu_{\Sigma_{\alpha}}=\mu_{\Sigma_{\tilde{\alpha}}}, \quad \mu_{\hat{\Sigma}_{\alpha}}=\mu_{\bar{\Sigma}_{\tilde{\alpha}}}, \\
& M_{\Sigma_{\alpha}}=M_{\Sigma_{\tilde{\alpha}}}, \quad M_{\Sigma_{\alpha}}=M_{\Sigma_{\tilde{\alpha}}}, \quad M_{\Sigma_{\alpha}^{*}}=M_{\tilde{\Sigma}_{\tilde{\alpha}}^{*}}\left({ }^{20}\right) .
\end{aligned}
$$

Appare invece dalle (31), (42) che, in generale è $m_{\alpha} \neq m_{\tilde{\alpha}}, \mu_{\widehat{\Sigma}_{\alpha}} \neq \mu_{\widehat{\Sigma}_{\tilde{\alpha}}}$.

\section{7. - Alcuni risultati.}

Le considerazioni svolte nei numeri precedenti consentono ora di stabilire alcuni risultati.

${ }^{(20)}$ Al risultato si può anche giungere sfruttando le relazioni ottenute ai numeri precedenti. Dalle (29), (30), (35), (36) discendono subito quattro delle uguaglianze (55). Tenuto conto di queste, dalle $(38),(40),(52)$ seguono le altre. 
Sussistono i Teoremi

$\mathbf{T}_{3}-$ Le medie $\mu_{\Sigma_{\alpha}}, M_{\Sigma_{\alpha}}, \mu_{\Sigma_{\alpha}}, M_{\Sigma_{\alpha}}$, introdotte ai $\mathrm{n} .10,11$ sono linearmente dipendenti. Precisamente risulta

$$
M_{\Sigma_{\alpha}}-\mu_{\Sigma_{\alpha}}=\left(M_{\Sigma_{\alpha}}-\mu_{\Sigma_{\alpha}}\right) \sin ^{2} \delta_{\alpha}
$$

$\mathbf{T}_{4}-$ La curvatura mista $\chi_{x \tilde{x}}$ si esprime linearmente nelle medie $\mu_{\Sigma_{\alpha}}, M_{\Sigma_{\alpha}}, \mu_{\Sigma_{\alpha}}, M_{\Sigma_{\alpha}}$. Precisamente

$$
\chi_{\alpha \tilde{\alpha}}=\left(2 M_{\Sigma_{\alpha}}-\mu_{\Sigma_{\alpha}}\right)+\left(2 M_{\Sigma_{\alpha}}-\mu_{\Sigma_{\alpha}}^{*}\right) \sin ^{2} \delta_{\alpha} .
$$

Per stabilire $\mathbf{T}_{3}$ basta notare che i secondi membri delle (29), (30), (35), (36), riguardati come forme lineari nelle variabili

$$
K_{u \tilde{u}}+K_{v \tilde{v}}, \quad K_{u \tilde{v}}+K_{v \tilde{u}}, \quad \chi_{u \tilde{u}, v \bar{v}}, \quad \chi_{u \tilde{v}, v \tilde{u}}
$$

risultano dipendenti.

Eliminando $K_{u \tilde{u}}+K_{v \tilde{v}}$ e $K_{u \tilde{v}}+K_{v \tilde{u}}$ tra le equazioni (29), (30), (36) e tenendo conto della (24) del n. 9, si perviene all'eguaglianza

$$
\chi_{\alpha \tilde{\alpha} \tilde{\alpha}}=3 M_{\Sigma_{\alpha}}-2 \mu_{\Sigma_{\alpha}}+M_{\Sigma_{\alpha}} \sin ^{2} \delta_{\alpha}
$$

dalla quale, in virtù della (56), segue subito la (57) e quindi il Teorema $\mathbf{T}_{\mathbf{4}}$.

In relazione poi alle curvature semplici sussistono i Teoremi

$\mathbf{T}_{5}-$ La curvatura semplice $K_{\alpha}$ è combinazione lineare delle medie delle curvature semplici relative ai sistemi $\Sigma_{\alpha}, \hat{\Sigma}_{\alpha}, \hat{\Sigma}_{\alpha}$ e della media $m_{x}$ introdotta al $\mathrm{n}$. 10. Precisamente

$$
K_{\alpha}=\left(2 \mu_{\widehat{\Sigma}_{\alpha}}-\mu_{\Sigma_{\alpha}^{*}}\right) \sin ^{2} \delta_{\alpha}-\mu_{\Sigma_{\alpha}} \cos ^{2} \delta_{\alpha}+2 m_{\alpha} \cos \delta_{\alpha} .
$$

$\mathbf{T}_{6}$ - La media aritmetica delle curvature $K_{\alpha}$ e $K_{\tilde{\alpha}}$ si esprime linearmente nelle medie relative ai sistemi $\Sigma_{\alpha}, \Sigma_{\alpha}^{*}, \Sigma_{\alpha}$. Precisamente risulta

$$
\frac{1}{2}\left(K_{\alpha}+K_{\tilde{\alpha}}\right)=\left(\mu_{\Sigma_{\alpha}}-\mu_{\Sigma_{\alpha}} \sin ^{2} \delta_{\alpha}\right)-2\left(3 M_{\Sigma_{\alpha}}-2 \mu_{\Sigma_{\alpha}}\right) \sin ^{2} \delta_{\alpha} .
$$

La (59) segue immediatamente dalla (42) del n. 13. L'eliminazione di $m_{\alpha}+m_{\tilde{\alpha}}$ tra le $(40),(38)$ conduce poi alla

$$
\frac{1}{4}\left(K_{\alpha}+2 \chi_{\alpha \tilde{\alpha}}+K_{\tilde{\alpha}}\right)=2 M_{\Sigma_{\alpha}}-\mu_{\Sigma_{\alpha}}-\left(3 M_{\Sigma_{\alpha}}-2 \mu_{\Sigma_{\alpha}}\right) \sin ^{2} \delta_{\alpha} .
$$

Da questa, in virtù delle $(56),(57)$ segue subito la $(60)$. 


\section{8. - Relaxioni tra medie.}

Un primo esempio è costituito dalla relazione (56) (Teorema $\mathbf{T}_{3}, \mathrm{n} .17$ ). Altri risultati sono questi.

$\mathbf{T}_{z}-$ Per le medie relative ai sistemi $\Sigma_{\alpha}, \hat{\Sigma}_{\alpha}, \hat{\Sigma}_{\alpha}, \hat{\Sigma}_{\alpha}$ sussistono le relazioni indipendenti

$$
\begin{aligned}
& 5\left(M_{\Sigma_{\alpha}}-\mu_{\Sigma_{\alpha}}\right)=\left(5 \mu_{\Sigma_{\alpha}}-7 M_{\Sigma_{\alpha}}-4 \mu_{\widehat{\Sigma}_{\alpha}}\right) \sin ^{2} \delta_{\alpha} . \\
& \left(M_{\Sigma_{\alpha}}-\mu_{\Sigma_{\alpha}}\right)\left(3-2 \sin ^{2} \delta_{\alpha}\right)= \\
& \quad=\left\{2 \mu_{\Sigma_{\alpha}}\left(1+\sin ^{2} \delta_{\alpha}\right)-M_{\Sigma_{\alpha}}\left(3+2 \sin ^{2} \delta_{\alpha}\right)-\left(\mu_{\mathcal{\Sigma}_{\alpha}}+\mu_{\widehat{\Sigma}_{\tilde{\alpha}}}\right)\right\} \sin ^{2} \delta_{\alpha} .
\end{aligned}
$$

$\mathbf{T}_{8}-$ La media aritmetica di $m_{\alpha}$ e $m_{\tilde{\alpha}}$ (n. 10) si esprime linearmente nelle medie delle curvature (semplici e miste), relative ai sistemi $\Sigma_{\alpha}, \stackrel{\circ}{\alpha}_{\alpha}$ di 2-spazi caratteristici. Si ha precisamente

$$
\left(m_{\alpha}+m_{\tilde{\alpha}}\right) \cos \delta_{\alpha}=\left(2 \mu_{\Sigma_{\alpha}} \sin ^{2} \delta_{\alpha}-\mu_{\Sigma_{\alpha}}\right) \cos ^{2} \delta_{\alpha}-\left(M_{\Sigma_{\alpha}} \sin ^{2} \delta_{\alpha}-M_{\Sigma_{\alpha}}\right)\left(1+2 \cos ^{2} \delta_{\alpha}\right) .
$$

Per stabilire $\mathbf{T}_{7}$ si procede cosi.

Si denoti anzitutto con ( $\widetilde{42}$ ) l'uguaglianza analoga alla (42), relativa però ad $\tilde{\alpha}$, tenendo conto anche delle osservazioni del n. 16. Si considerino poi le uguaglianze (40), (38), (42), ( $\widetilde{42}),(52)$, sostituendo in esse a $\chi_{\alpha \tilde{\alpha}}$ l'espressione a secondo membro della (57).

Cio premesso si prova che le forme lineari nelle variabili $m_{\alpha}, m_{\tilde{\alpha}}, K_{\alpha}, K_{\tilde{\alpha}}$, che figurano nelle accennate uguaglianze, sono dipendenti. Anzi, poichè il rango della corrispondente matrice è 3 , si possono determinare tra queste forme lineari due relazioni indipendenti.

Per questa via, utilizzando prima le $(40),(38),(52)$, poi le $(40),(38),(42),(\widetilde{42})$, e tenendo conto anche della (56), si perviene, rispettivamente, alle relazioni (62) e (63) del Teorema $\mathbf{T}_{7}$.

La dimostrazione di $\mathbf{T}_{8}$ è più semplice. Basta infatti eliminare tra le (38), (40) l'espressione $K_{\alpha}+2 \chi_{\tilde{\alpha} \tilde{\alpha}}+K_{\tilde{\alpha}}$, per giungere alla (64).

Conviene ancora aggiungere una osservazione. Dalla accennata dimostrazione di $\mathbf{T}_{7}$ risulta che ogni altra relazione lineare nelle medie relative ai sistemi $\Sigma_{\alpha}, \hat{\Sigma}_{\alpha}, \hat{\Sigma}_{\alpha}, \hat{\Sigma}_{\alpha} \dot{e}$ una combinazione lineare delle (62), (63). In particolare, eliminando tra queste $M_{\Sigma_{\alpha}}-\mu_{\Sigma_{*}}$, si ottiene la relazione

$$
\left(6 M_{{\underline{Y_{\alpha}}}_{\alpha}}-5 \mu_{\mathfrak{\Sigma}_{\alpha}}\right)\left(1-4 \sin ^{2} \delta_{\alpha}\right)=5\left(\mu_{\widehat{\Sigma}_{\alpha}}+\mu_{\widehat{\Sigma}_{\bar{z}}}\right)-4 \mu_{\hat{\Sigma}_{\alpha}}\left(1+2 \cos ^{2} \delta_{\alpha}\right),
$$

utile nel seguito. 


\section{9. - Casi particolari.}

Si consideri dapprima l'ipotesi che il 2-spazio $\alpha$ sia a prodotto hermitiano reale (n. 3). Risulta allora $\delta_{\alpha}=\pi / 2$ (n. 4) e pertanto le espressioni delle medie $\mu, M$, ottenute nei n. 10, 11, 12, 13, 14 si semplificano. Lo stesso accade per le uguaglianze dei n. 17, 18.

Conviene sottolineare due risultati

$\mathbf{T}_{9}-$ La curvatura $K_{\alpha}$ di un 2-spazio $\propto$ p.h.r. si esprime linearmente nelle medie delle curvature semplici relative ai sistemi di 2 -spazi p.h.r. $\hat{\Sigma}_{\alpha}, \hat{\Sigma}_{\alpha}$. Preoisamente

$$
K_{\alpha}=2 \mu_{\widehat{\Sigma}_{\alpha}}-\mu_{\Sigma \alpha}^{*} .
$$

$\mathbf{T}_{10}-S e \propto \grave{e}$ p.h.r., le medie delle ourvature miste, relative ai sistemi di 2-spazi caratteristici $\Sigma_{\alpha}, \stackrel{\Sigma}{\alpha}_{\alpha}$, sono uguali. Ciò̀

$$
M_{\Sigma_{\alpha}}=M_{\Sigma_{\alpha}} .
$$

Le (66), (67) seguono subito dalle (59), (64).

Anche se, a partire dal n. 10, esclusa esplicitamente dalle considerazioni, conviene ora, per completezza, esaminare l'ipotesi che il 2-spazio $\alpha$ sia caratteristico (n. 2). Si vede subito, allora, che i sistemi $\Sigma_{\alpha}, \hat{\Sigma}_{\alpha}, \hat{\Sigma}_{\alpha}, \hat{\Sigma}_{\alpha}, \hat{\Sigma}_{\alpha}$ degenerano nel 2 -spazio $\alpha$ stesso oppure perdono significato.

Le relazioni dei $\mathrm{n} .10,11,12,13,14$, concernenti le medie $\mu, M, m$, si riducono nell'ipotesi attuale $\left(\delta_{\alpha}=0\right)$ a banali identità ( $\left.{ }^{21}\right)$. Lo stesso avviene per le uguaglianze dei n. 15, 17, 18, salvo per la (65) che perde senso ${ }^{22}$ ).

\section{0. - Isotropia in media e $J$-isotropia.}

Per le varietà quasi hermitiane $V$ si possono considerare, in ogni punto $x$, le ipotesi di isotropia e di isotropia caratteristica ${ }^{\left({ }^{3}\right)}$. Nel primo caso si richiede che, per ogni 2 -spazio $\propto$ di $\tau$, la curvatura $K_{\alpha}$ sia costante; nel secondo si richiede soltanto che $K_{\alpha}$ sia costante sui 2-spazi caratteristici.

Esistono però altre possibilità interessanti. Si può ad esempio richiedere che, per ogni $\alpha$ di $\tau$, sia costante la media aritmetica di $K_{\alpha}$ e $K_{\tilde{\alpha}}$ (isotropia in media), oppure che sia costante la curvatura mista $\chi_{\alpha \tilde{x}}(J$-isotropia).

$\left({ }^{21}\right)$ Occorre preventivamente seriverle nella forma originaria, eliminando le eventuali potenze negative di $\sin \delta_{\alpha}$. Conviene inoltre ragionare sulla (33) anzichè sull'equivalente (36).

${ }^{(22)}$ Per la (54) del n. 15 conviene preventivamente moltiplieare membro a membro per

$$
\sin ^{2} \delta_{\alpha}=\sin ^{2} u \tilde{v}=\sin ^{2} v \tilde{u}=\sin ^{2} x \tilde{y}=\sin ^{2} y \tilde{x},
$$

- far intervenire il tensore $R$ di Riemann (n. 9).

${ }^{(23)}$ Per il caso particolare delle varietà kähleriane si veda p. es. G. B. Rrzza [8], n. 6. 
Conviene notare subito che l'isotropia implica l'isotropia in media e che tanto l'isotropia in media quanto la J-isotropia implicano l'isotropia caratteristica ( $\left.{ }^{24}\right)$.

Sussistono poi i teoremi

$\mathbf{T}_{11}-$ Se in un punto $x$ di $V$ si ha isotropia in media, sussistono le uguaglianze

$$
\begin{gathered}
\mu_{\Sigma_{\alpha}}=\mu_{\Sigma_{\alpha}}=\mu_{\dot{\Sigma}_{\alpha}}=\frac{1}{2}\left(\mu_{\tilde{\Sigma}_{\alpha}}+\mu_{\hat{\Sigma}_{\tilde{\alpha}}}\right)=\mu_{\hat{\Sigma}_{\alpha}}=c, \\
M_{\Sigma_{\alpha}}=\frac{c}{2}\left(1+\cos ^{2} \delta_{\alpha}\right), \quad M_{\Sigma_{\alpha}}=M_{\Sigma_{\alpha}}=\frac{0}{2}, \quad \frac{1}{2}\left(m_{\alpha}+m_{\tilde{\alpha}}\right)=c \cos \delta_{\alpha},
\end{gathered}
$$

dove $c$ è la costante di isotropia.

$\mathbf{T}_{12}-$ Se in un punto $x$ di $V$ si ha $J$-isotropia, sussistono le uguaglianze

$$
\begin{array}{ll}
\mu_{\Sigma_{\alpha}}=\mu_{\Sigma_{\alpha}}=\mathrm{c}, & \mu_{\Sigma_{\alpha}}^{*}=-2 c, \\
M_{\Sigma_{\alpha}}=\frac{e}{2}\left(2+\sin ^{2} \delta_{\alpha}\right), & M_{\Sigma_{\alpha}}=-\frac{3}{2} c,
\end{array}
$$

dove c è la costante di isotropia.

$\mathbf{T}_{13}-$ Se in un punto $x \not i V$ si ha isotropia in media, risulta

$$
\chi_{\alpha \tilde{x}}=c \cos ^{2} \delta_{\alpha},
$$

dove o è la costante di isotropia.

I teoremi ora enunciati asseriscono in sostanza che, in ipotesi di isotropia in media e di $J$-isotropia, molte delle medie considerate in questo lavoro risultano costanti. Le espressioni $M_{\Sigma_{\alpha}}, \frac{1}{2}\left(m_{\alpha}+m_{\tilde{\alpha}}\right), \chi_{\alpha \tilde{\alpha}}$ dipendono invece dalla deviazione caratteristica $\delta_{\alpha}$ del 2-spazio in considerazione ed assumono i valori estremi in corrispondenza a $i$ 2-spazi caratteristici $\left(\delta_{\alpha}=0\right)$ e p.h.r. $\left(\delta_{\alpha}=\pi / 2\right)$.

Se in un punto $x$ di $V$ si ha addirittura isotropia, sussistono naturalmente le uguaglianze di $\mathbf{T}_{12}$ e la (68) di $\mathbf{T}_{\mathbf{1 3}}$. Si può anzi aggiungere che

$$
\mu_{\widehat{\Sigma}_{\alpha}}=c, \quad m_{\alpha}=e \cos \delta_{\alpha} .
$$

\section{1. - Dimostrazioni.}

Per stabilire i Teoremi $\mathbf{T}_{11}$ e $\mathbf{T}_{13}$ si procede così. L'ipotesi è che, per ogni $\alpha$ di $\tau$ sia

$$
\frac{1}{2}\left(K_{\alpha}+K_{\tilde{a}}\right)=e .
$$

${ }^{(24)}$ Se $\alpha$ è caratteristica, $\alpha=\tilde{\alpha}$, onde $K_{\tilde{\alpha}}=\chi_{\tilde{\alpha} \tilde{\alpha}}=\chi_{\alpha \tilde{\alpha}}=\chi_{\alpha \alpha}=K_{\alpha}$. 
Poichè, come si è notato, l'ipotesi implica isotropia caratteristica, segue subito l'asserto per $\mu_{\Sigma_{\alpha}}$ e $\mu_{\Sigma_{\alpha}}$. Per valutare $\mu_{\hat{\Sigma}_{\alpha}}+\mu_{\hat{\Sigma}_{\tilde{\alpha}}}$, si utilizzano le (14), $(5),($ n. 7,5$)$ con riferimento ad $\alpha$ e ad $\tilde{\alpha}$.

Assunta in $\tilde{\alpha}$ come base ortonormale $\tilde{u}, \tilde{v}$ si riconosce subito che la coppia di parametri $\varphi, \psi$ che individua il 2-spazio generico $\varrho$ di $\hat{\Sigma}_{\alpha}$ (n. 13), dà luogo nel secondo caso al 2-spazio $\tilde{\varrho}$ di $\hat{\Sigma}_{\tilde{\alpha}}$. Si ha allora

$$
\mu_{\widehat{\Sigma}_{\alpha}}+\mu_{\widehat{\Sigma}_{\tilde{\alpha}}}=\frac{1}{(2 \pi)^{2}} \int_{0}^{2 \pi} d \varphi \int_{0}^{2 \pi} K_{\varrho} d \psi+\frac{1}{(2 \pi)^{2}} \int_{0}^{2 \pi} d \varphi \int_{0}^{2 \pi} K_{\tilde{0}} d \psi=\frac{1}{(2 \pi)^{2}} \int_{0}^{2 \pi} d \varphi \int_{0}^{2 \pi}\left(K_{\varrho}+K_{\tilde{0}}\right) d \psi=20
$$

Lo stesso ragionamento si conduce per $\mu_{\Sigma_{\alpha}}$ e per $\mu_{\vec{\Delta} \alpha}$ notando che, in virtù della (55) del n. 16, risulta $\mu_{\Sigma_{\alpha}}=\mu_{\Sigma_{\tilde{\alpha}}}, \mu_{\vec{\Sigma}_{\alpha}}=\mu_{\Sigma_{\tilde{\alpha}}}\left({ }^{25}\right)$

A questo punto, dalla (60) si ricava $M_{\Sigma_{\infty}}$; successivamente, dalle (62), (64) si ottengono $M_{\Sigma_{\alpha}}$ ed $m_{\alpha}$. Infine, dalle $(56),(57)$ si ricavano $M_{\Sigma_{\alpha}}$ e $\chi_{\alpha \tilde{\alpha}}$.

Per stabilire il Teorema $\mathbf{T}_{12}$, si ricordi che l'ipotesi

$$
\chi_{x \tilde{x}}=c
$$

per ogni $\alpha$, implica isotropia caratteristica; di qui segue l'asserto per $\mu_{\Sigma_{\alpha}}$ e $\mu_{\Sigma_{\alpha}}$.

Dalla (24) del $\mathrm{n} .9$ segue $\chi_{u \tilde{u}, v \tilde{v}}=c\left(1+\sin ^{2} \delta_{\alpha}\right)$ e ciò consente di ricavare $M_{\Sigma_{\alpha}}$ dalla (30). Il sistema delle $(56),(57)$ conduce poi a $\mu_{\Sigma \infty}^{*}$ ed a $M_{\Sigma_{\alpha}}$.

Si noti infine che, nell'ipotesi di isotropia, considerata alla fine del n. 20 la prima delle (69) è immediata; la seconda segue subito dalla (59) del Teorema $\mathbf{T}_{\breve{s}}$.

\section{2. - Teoremi tipo Schur.}

In questo numero si mostra come condizioni locali di "isotropia possano dare indicazioni di natura globale. Precisamente sussiste il teorema

$\mathbf{T}_{14}-$ Se in ogni punto $x$ di $V$ si ha isotropia in media e $J$-isotropia, allora le costanti di isotropia (a priori dipendenti da $x$ ) sono entrambe nulle.

$\mathbf{E}$ interessante anche il corollario

$\mathbf{C}_{1}-S e$ in ogni punto $x d i V$, si ha isotropia e J-isotropia, $V$ è localmente euclidea.

Le ipotesi del Teorema $\mathbf{T}_{14}$ possono essere attenuate in virtù di questo risultato

$\mathbf{T}_{15}-$ Se in un punto $x$ di $V$, la media aritmetica di $K_{\alpha}$ e $K_{\tilde{\alpha}}$ e la curvatura mista $\chi_{\alpha \tilde{\alpha}}$ sono costanti per ogni $\alpha$ caratteristico o p.h.r., si ha isotropia in media e J-isotropia, anzi la media e la curvatura considerate sono entrambe nulle per ogni $\alpha$.

${ }^{(25)}$ Il risultato relativo a $\mu_{\Sigma_{\alpha}}$ può anche dedursi direttamente dalla (54) del n. 15. 
L'ipotesi del Teorema $\mathbf{T}_{14}$ è

$$
\frac{1}{2}\left(K_{\alpha}+K_{\tilde{\alpha}}\right)=c_{1}, \quad \chi_{\alpha \tilde{x}}=c_{2},
$$

con $c_{1}, c_{2}$ costanti per ogni $\alpha$. Se in particolare $\alpha$ è caratteristico $(\alpha=\tilde{\alpha})$ risulta $c_{1}=c_{2}=c$. Confrontando $\mathrm{i}$ risultati dei Teoremi $\mathbf{T}_{11}$ e $\mathbf{T}_{12}$, segue subito $c=0$ e cioè l'asserto $\left({ }^{26}\right)$.

Nell'ipotesi di $\mathbf{C}_{1}$, denotata con $c_{1}$ la costante di isotropia, poichè $\frac{1}{2}\left(K_{\alpha}+K_{\tilde{\alpha}}\right)=$ $=K_{\alpha}=c_{1}$, dal Teorema $\mathbf{T}_{14}$ segue $K_{\alpha}=0$ per ogni $\alpha$, cioè l'asserto.

Per stabilire il Teorema $\mathbf{T}_{1.5}$ si procede così. Per ipotesi sussistono le (72) con $\alpha$ caratteristico ovvero a prodotto hermitiano reale. In particolare, se $\alpha$ è caratteristico $(\alpha=\tilde{\alpha})$, risulta $c_{1}=c_{2}=c$; si ha pure $\mu_{\Sigma_{\alpha}}=\mu_{\Sigma_{\alpha}}=c$, i sistemi $\Sigma_{\alpha}, \Sigma_{\alpha}^{\circ}$ essendo costituiti da 2-spazi caratteristici. Si utilizzi ora la prima delle (72) con riferimento ai 2-spazi p.h.r. di $\hat{\Sigma}_{\alpha}$ e di $\stackrel{\Delta}{\alpha}_{\alpha}$; il ragionamento sviluppato al n. 21 nella dimostrazione del Teorema $\mathbf{T}_{11}$ porta a concludere che $\mu_{\Sigma_{\alpha}}=\mu_{\hat{\Sigma}_{\alpha}}=e$.

Ciò premesso, dalla (60) segue subito che $M_{\Sigma_{\alpha}}=e / 2$ sui 2 -spazi p.h.r.; in virtù della (67) del Teorema $\mathbf{T}_{10}$ si ha $M_{\Sigma_{\alpha}}=c / 2$ per ogni $\alpha$ p.h.r.

D'altra parte, utilizzando la seconda delle (72) con riferimento ai 2-spazi p.h.r., dalle (24) del n. 9 segue che $\chi_{u \tilde{u}, v \tilde{v}}=2 e$, per ogni $\alpha$ (con base ortonormale $u, v$ ) a prodotto hermitiano reale. Dalla (30) del n. 10 discende subito $M_{\Sigma_{\alpha}}=\frac{3}{2} c$ per ogni $\alpha$ p.h.r. Il confronto col risultato precedentemente ottenuto permette di concludere che $c=0$.

Si considerino dunque le (72) con $c_{1}=c_{2}=0$ ed $\alpha$ caratteristico o p.h.r. Dalla (29) del n. 10 segue $\chi_{u \tilde{u}, v \tilde{v}}=0$ per ogni $\alpha$ (con base ortonormale $\left.u, v\right)\left({ }^{27}\right)$.

Dalle (30), (36) segue $M_{\Sigma_{\alpha}}=M_{\Sigma_{\alpha}^{*}}=0$ per ogni $\alpha$ e poichè, come si è visto, è anche $\mu_{\Sigma_{\alpha}}=\mu_{\Sigma_{\alpha}}^{*}=0$, dalla $(57)$ del $\mathrm{n}$. 17 segue subito $\chi_{\alpha \tilde{\alpha}}=0$ per ogni $\alpha$.

Infine, tenuto conto dei risultati già ottenuti, dalla (62) del n. 18 deriva $M_{\Sigma_{\alpha}}=0$ per ogni $\alpha$; successivamente, dalla $(60)$ segue $\frac{1}{2}\left(K_{\alpha}+K_{\tilde{\alpha}}\right)=0$ per ogni $\alpha$. II Teorema $\mathbf{T}_{15}$ è dunque dimostrato.

\section{3. - Caso kähleriano.}

Si consideri ora il caso particolare in cui $V$ sia una varietà kähleriana $\left({ }^{28}\right)$.

Poichè, come è noto $\left.{ }^{29}\right)$, in questa ipotesi, per ogni quanterna di vettori $u, v, x, y$ di $\tau$ risulta

$$
R(u v x y)=R(u v \tilde{x} \tilde{y})
$$

(26) Alla stessa conclusione si perviene confrontando la (71) con la (68) del teorema $\mathbf{T}_{13}$.

(27) I 2-spazi $u \tilde{v}$ e vĩ sono p.h.r. (n. 3) e corrispondenti nella $J$.

(28) Per le generalità sulle varietà kähleriane vedi p. es. K. YANo [11], ch. 4; S. KoBAYASHI - K. NOMTzU [4], II, p. 149-178.

$\left({ }^{29}\right)$ Vedi p. es. K. YaNo [11], (1.10), p. 71; S. KobaYaShI - K. NomizU [4], II, p. 165. 
tenute presenti le classiche proprietà del tensore $R$ di Rremann, le definizioni (21), (20) del n. 9 e la (1) del n. 3, si perviene subito alla relasione

$$
\chi_{\alpha \beta}=\chi_{\alpha \tilde{\beta}}=\chi_{\tilde{\alpha} \beta}=\chi_{\tilde{\alpha} \tilde{\beta}}
$$

in particolare, se $\beta=\alpha$, si ottiene la relazione

$$
K_{x}=\chi_{\alpha \tilde{\alpha}}=K_{\tilde{\alpha}} .
$$

Cio premesso, conviene segnalare qualche risultato.

$\mathbf{T}_{16}-S e V$ è una varietà kähleriana, le medie $m_{\alpha}$ e $\mu_{\widehat{\Sigma}_{\alpha}}(\mathrm{n} .10,13)$ sono J-invarianti, ciò̀

$$
m_{\alpha}=m_{\tilde{\alpha}}, \quad \mu_{\widehat{\Sigma}_{\alpha}}=\mu_{\widehat{\Sigma}_{\tilde{\alpha}}},
$$

e la media $M_{\mathcal{E}_{\alpha}}$ delle curvature miste dei 2-spazi p.h.r. del sistema $\dot{\Sigma}_{\alpha}$ del $\mathrm{n} .11$ è nulla.

Il primo risultato completa nell'ipotesi attuale le ultime considerazioni del n. 16; il secondo va invece avvicinato ai Teorema $\mathbf{T}_{1}, \mathbf{T}_{2}$ relativi anch'essi a sistemi di 2 -spazi p.h.r. (n. 13, 14).

Aneora, sulle varietà kähleriane, la curvatura $K_{\alpha}$ di un qualunque 2 -spazio $\alpha$ si esprime in modo estremamente semplice in funzione delle curvature (sempliei e miste) dei 2-spazi caratteristici di $\Sigma_{\alpha}$, ovvero in funzione delle curvature semplici dei 2-spazi (rispettivamente caratteristici e p.h.r.) dei sistemi $\Sigma_{\alpha}$ e $\dot{\Sigma}_{\alpha}$ : Precisamente risulta

$\mathbf{T}_{17}-$ Se $V$ è una varietà kähleriana, per ogni 2-spazio $\alpha$ di $\tau$ sussiste la relazione

$$
K_{\alpha}=3 M_{\Sigma_{\alpha}}-2 \mu_{\Sigma_{\alpha}}=\mu_{\Sigma_{\alpha}}-3 \mu_{\Sigma_{\alpha}} \sin ^{2} \delta_{\alpha}
$$

La prima uguaglianza è nota $\left({ }^{30}\right)$.

Infine, nell'ipotesi attuale, le medie $M_{\Sigma_{\alpha}}, M_{\widehat{\Sigma}_{\alpha}}, M_{\hat{\Sigma}_{\alpha}}$ relative alle curvature miste, sono nulle (Teoremi $\mathbf{T}_{16}, \mathbf{T}_{1}, \mathbf{T}_{2}$ ); le medie $\mu_{\Sigma_{\alpha}}, \mu_{\widehat{\Sigma}_{\alpha}}, \mu_{\widehat{\Sigma}_{\alpha}}$, relative alle curvature semplici intervengono invece nel Teorema

$\mathbf{T}_{18}-$ Se $V_{\Delta} \grave{e}$ una varietà kähleriana, per le medie $\mu_{\Sigma_{\alpha}}, \mu_{\widehat{\Sigma}_{\alpha}}, \mu_{\hat{\Sigma}_{\alpha}}$, concernenti $i$ siste$m i \dot{\Sigma}_{\alpha}, \hat{\Sigma}_{\alpha}, \hat{\Sigma}_{\alpha}$, di 2-spazi p.h.r., risulta

$$
\begin{aligned}
& \mu_{\Sigma_{\alpha}} \sin ^{2} \delta_{\alpha}=\mu_{\Sigma_{\alpha}}-M_{\Sigma_{\alpha}}, \\
& \mu_{\widehat{\Sigma}_{\alpha}} \sin ^{2} \delta_{\alpha}=\left(\mu_{\Sigma_{\alpha}}-M_{\Sigma_{\alpha}}\right) \cos ^{2} \delta_{\alpha}+\left(\mu_{\varepsilon_{\alpha}}-M_{\Sigma_{\alpha}}\right) \sin ^{4} \delta_{\alpha}, \\
& \mu_{\Sigma_{\alpha}} \sin ^{2} \delta_{\alpha}=\frac{3}{4}\left(\mu_{\Sigma_{\alpha}}-M_{\Sigma_{\alpha}}\right)+\frac{1}{4}\left(\mu_{\Sigma_{\alpha}}-M_{\Sigma_{\alpha}}\right) \sin ^{2} \delta_{\alpha} .
\end{aligned}
$$

$\left({ }^{30}\right)$ G. B. R R RZA [8], p. 85. 
Sussiste quindi la relazione

$$
\mu_{\widehat{\Sigma}_{\alpha}}-\mu_{\Sigma_{\alpha}}=4\left(\mu_{\widehat{\Sigma}_{\alpha}}-\mu_{\Sigma_{\alpha}}\right) \sin ^{2} \delta_{\alpha} .
$$

Per stabilire $\mathbf{T}_{16}$, si osservi che, in virtù della (73), dalle (31), (30) del n. 10 seguono immediatamente la prima delle (75) ed $M_{\Sigma_{\alpha}}=0$. La seconda delle (75) si ottiene poi facilmente, ricordando una osservazione del n. 21 e tenendo presente la (74).

Dalle (57), (56), a causa della (74) e del fatto che, come si è visto $M_{\Sigma_{x}^{*}}=0$, si deduce poi la (76) del Teorema $\mathbf{T}_{17}$.

Infine, nel Teorema $\mathbf{T}_{18}$, la prima uguaglianza scende subito dalla (56). Per le due successive si osservi anzitutto che, in virtù delle (74), (76), la (61) del n. 17 diviene

$$
\mu_{\Sigma_{\alpha}}-M_{\Sigma_{\alpha}}=\left(3 M_{\Sigma_{\alpha}}-2 \mu_{\Sigma_{x}}\right) \sin ^{2} \delta_{\alpha}
$$

l'asserto segue facilmente dalle (63), (62) del n. 18, tenendo presente la (78). La (77) poì è immediata conseguenza delle tre uguaglianze che la precedono.

I risultati enunciati in questo numero sono ora dimostrati.

Conviene ancora aggiungere qualche osservazione.

Per le varietà kähleriane le relazioni (53), (30) (n. 15, 10), che esprimono le medie $\mu_{\Sigma_{x}}$, $M_{\Sigma_{\alpha}}$ in funzione delle curvature (semplici e miste) di quattro 2-spazi earatteristici di $\Sigma_{\alpha}$, sono note $\left({ }^{31}\right)$. In altri termini, in questo lavoro si riconosce che le formule note nel caso kähleriano restano inalterate passando al caso più generale della varietà quasi hermitiane.

Si noti infine che, a norma della (74), sulle varietà kähleriane l'isotropia in media e la $J$-isotropia (introdotte al $\mathrm{n} .20$ ) equivalgono all'isotropia. Di conseguenza, il Teorema $\mathbf{T}_{\mathbf{1 4}}$ e il corollario $\mathbf{C}_{1}$ si riducono ad un noto teorema di $\mathbf{S}$. BochNer $\left({ }^{32}\right)$.

Il Teorema $\mathbf{T}_{\mathbf{1 5}}$ consente poi di attenuare ulteriormente l'ipotesi del teorema ora accennato. Precisamente

$\mathbf{T}_{19}-$ Se in ogni punto $x$ di una varietà kähleriana $V$ la curvatura $K_{\alpha} \dot{e}$ costante per ogni 2-spazio \& caratteristico o a prodotto hermitiano reale, allora $V$ è localmente euclidea.

(31) G. B. Rizza [8], p. 86 .

${ }^{32}$ Vedi p. es. K. Yano - S. Bochner [12]. p. 129.

\section{BIBLIOGRAFIA}

[1] E. Bomerant, spazi riemanniani luoghi di varietà totalmente geodetiche, Rend. Circ. Mat. Palermo, 48 (1924).

[2] M. BruNi, Bu alcuni sistemi di sottospasi di uno spazio hermitiano, Rend. Mat. Roma, $20(1961)$. 
[3] S. 1. GoLDBERG - S. KOBAYASH, Holomorphie bisectional ourvature, J. Differential Geometry, 1 (1967).

[4] S. Kobayashi - K. Nomizu, Foundation of differential Geometry, I \& II, interscience Publ., New York, 1963 e 1969.

[5] E. Martmelci, Metrica hermitiana e metrica euelidea e simplettica associate, Rend. Mat. Roma, 6, 2 (1969).

[6] G. B. RrzzA, Deviazione caratteristica delle faccetle piane di una varietà a struttura complessa, Rend. Lincei, 8, 24 (1958).

[7] G. B. RIzzA, Deviazione caratteristica e proprietà locali delle 2q-jaccette di una $V_{2 n}$ a struttura complessa, Rend. Acc. Naz. dei XL, 4, 10 (1959).

[8] G. B. RizzA, Sulla curvatura delle faccette di una varietà kähleriana, Rend. Lincei, 47 (1959).

[9] G. B. RIzzA, Sulle connessioni di una varietà quasi complessa, Ann. di Mat., 6, 68 (1965).

[10] J. A. Schouten, Ricci Caloulus, Springer, Berlin, 1954.

[11] K. YANo, Differential Geometry on complex and almost complex spaces, Pergamon Press, Oxford, 1965.

[12] K. Yano - S. Bochner, Curvature and Betti numbers, Ann. of Math. Studies n. 32, Princeton Univ. Press, 1953. 